\title{
Momentum dependent mean-field dynamics of compressed nuclear matter and neutron stars
}

\author{
Theodoros Gaitanos* and Murat M. Kaskulov ${ }^{\dagger}$ \\ Institut für Theoretische Physik, Universität Giessen, D-35392 Giessen, Germany
}

(Dated: November 3, 2018)

\begin{abstract}
Nuclear matter and compact neutron stars are studied in the framework of the non-linear derivative (NLD) model which accounts for the momentum dependence of relativistic mean-fields. The generalized form of the energy-momentum tensor is derived which allows to consider different forms of the regulator functions in the NLD Lagrangian. The thermodynamic consistency of the NLD model is demonstrated for arbitrary choice of the regulator functions. The NLD approach describes the bulk properties of the nuclear matter and compares well with microscopic calculations and Dirac phenomenology. We further study the high density domain of the nuclear equation of state $(E o S)$ relevant for the matter in $\beta$-equilibrium inside neutron stars. It is shown that the low density constraints imposed on the nuclear EoS and by the momentum dependence of the Schrödinger-equivalent optical potential lead to a maximum mass of the neutron stars around $M \simeq 2 M_{\odot}$ which accommodates the observed mass of the J1614-2230 millisecond radio pulsar.
\end{abstract}

PACS numbers: 21.65.-f, 21.65.Mn, 25.40.Cm

\section{INTRODUCTION}

Relativistic hadrodynamics (RHD) of interacting nucleons and mesons provide a simple and successful tool for the theoretical description of different nuclear systems such as nuclear matter, finite nuclei, heavy-ion collisions and compact neutron stars [1]. Starting from the pioneering work of Duerr [2], simple RHD Lagrangians have been introduced $[3,4]$ and since then many different extensions of RHD approach, which rely on relativistic mean-field (RMF) approximation, have been developed. They describe the saturation mechanism in nuclear matter and generate a natural mechanism for the strong spin-orbit force in nuclei. An energy dependence of the Schrödinger-equivalent optical potential $[5,6]$ is thereby included as a consequence of a relativistic description. However, when using the standard RHD Lagrangian in RMF approximation, the nucleon selfenergies become simple functions of density only, and do not depend explicitly on momentum of the nucleon. As a consequence a linear energy dependence of the Schrödinger-equivalent optical potential with a divergent behavior at high energies arises [7]. This wellknown feature contradicts Dirac phenomenology $[5,6,8]$.

To solve this issue one may go beyond the mean-field approximation in a quantum field theoretical framework by a systematic diagrammatic expansion of nucleon selfenergies. For instance, in Dirac-Brueckner-Hartree-Fock (DBHF) [911] calculations the nucleon selfenergies indeed depend on both the density and single particle momentum. They reproduce the empirical saturation point of nuclear matter as well as the energy dependence of the optical potential at low energies. However, the DBHF approach has its apparent limitations at high energies and densities relevant, for instance, in heavy-ion collisions where its application within transport theory turns out to be intricate $[12,13]$. Also the thermodynamic consistency of the DBHF calculations is not obvious [14].

\footnotetext{
* theodoros.gaitanos@theo.physik.uni-giessen.de

† murat.kaskulov@theo.physik.uni-giessen.de
}

As an alternative approach to ab-initio DBHF calculations for the nuclear many-body systems a phenomenological treatment of the problem in the spirit of the RMF approximation is still considered as a powerful tool. However, the simple Lagrangian of RHD [3, 4] has to be further modified for a quantitative description of static nuclear systems such as nuclear matter and/or finite nuclei. Therefore, it is mandatory to introduce new terms, e.g., including non-linear self interactions of the scalar [15] and/or vector [16] meson fields, or to modify existing contributions in the Lagrangian, e.g., introducing density dependent meson-nucleon couplings [17-19]. The model parameters have to be then fitted to properties of nuclear matter and/or atomic nuclei, since, they cannot be derived in a simple manner from a microscopic description.

The momentum dependence of in-medium interactions becomes particularly important in description of nuclear collision dynamics such as heavy-ion collisions. Indeed, analyses of proton-nucleus scattering data $[5,6]$ show that the protonnucleus optical potential starts to level off already at incident energies of about $300 \mathrm{MeV}$. Thus, other RMF approaches have been developed by including additional non-local contributions, i.e., by introducing Fock-terms, on the level of the RMF selfenergies leading to a density and momentum dependent interactions [7]. However, such a treatment is not covariant and also its numerical realization in actual transport calculations is rather difficult [7]. Another approach has been proposed in [20] and more recently in [21, 22] by introducing higher order derivative couplings in the Lagrangian of RHD. In Ref. [20] such gradient terms have been studied with the conclusion of a softening of the nuclear EoS. In another study of Ref. [22] both the density dependence of the nuclear EoS and the energy dependence of the optical potential have been investigated. While the modified interactions of meson fields with nucleons explain the empirical energy dependence of the optical potential, a stiff EoS at high densities results from an introduction of an explicit density dependence of the nucleonmeson couplings with additional parameters. The impact of momentum dependent RMF models on nuclear matter bulk properties and particularly on the high density domain of EoS relevant for neutron stars is presently less understood. 
The purpose of the present work is to develop a relativistic and thermodynamically consistent RMF model, which provides the correct momentum dependence of the nucleon selfenergies and agrees well with available empirical information on nuclear matter ground state, in a self consistent Lagrangian framework. Some steps in this direction have been already done in Refs. [23-25] where the concept of non-linear derivative meson-nucleon Lagrangian has been introduced. However, the calculations of Refs. [23-25] were based on a particular exponential form of the regulators in the RHD Lagrangian and a detailed study of nuclear matter ground state properties has not been done. In the present work the generalized form of the energy-momentum tensor in the NLD model is derived and allows to consider different regulator functions in the Lagrangian. The thermodynamic consistency of the NLD model is demonstrated for arbitrary choice of the regulators. A thorough study of the properties of nuclear matter around saturation density is further performed. The model describes the bulk properties of the nuclear matter and compares well with microscopic calculations and Dirac phenomenology. We also investigate the high density region of the NLD EoS relevant for the neutron stars. It is found that the low density constraints imposed on the nuclear matter EoS and by the momentum dependence of the Schrödinger-equivalent optical potential lead to a maximum mass of the neutron stars around $M \simeq 2 M_{\odot}$. It is demonstrated that the high density pressure-density diagram as extracted from astrophysical measurements $[26,27]$ can be well described with nucleonic degrees of freedom only.

\section{FIELD THEORY WITH HIGHER DERIVATIVES}

The non-linear derivative (NLD) model is based on a fieldtheoretical formalism which accounts for the higher-order derivative interactions in the RHD Lagrangian. As a consequence, the conventional RHD mean-field theory based on minimal interaction Lagrangians has to be extended to the case of higher-order non-linear derivative functionals.

For that purpose we consider the most general structure of a Lagrangian density $\mathcal{L}$ with higher-order field derivatives, i.e.

$$
\mathcal{L}\left(\varphi_{r}(x), \partial_{\alpha_{1}} \varphi_{r}(x), \partial_{\alpha_{1} \alpha_{2}} \varphi_{r}(x), \cdots, \partial_{\alpha_{1} \cdots \alpha_{n}} \varphi_{r}(x)\right),
$$

where it is supposed that $\mathcal{L}$ has continuous derivatives up to order $n$ with respect to all its arguments, that is

$\partial_{\alpha_{1} \cdots \alpha_{n}} \varphi_{r}(x) \equiv \frac{\partial}{\partial x^{\alpha_{1}}} \cdots \frac{\partial}{\partial x^{\alpha_{n}}} \varphi_{r}(x) \equiv \partial_{\alpha_{1}} \cdots \partial_{\alpha_{n}} \varphi_{r}(x)$,

where $\alpha_{i}$ is a four index and $x$ denotes the coordinates in Minkowski space. The order $n$ can be a finite number or $n \rightarrow \infty$. The subscript $r$ denotes different fields, for instance, in the case of the spinor fields one would have $\varphi_{1}=\Psi$ and $\varphi_{2}=\bar{\Psi}$.

The derivation of the generalized Euler-Lagrange equations of motion follows from the variation principle for the action $S=\int d^{4} x \mathcal{L}(x)$ with the Lagrangian of Eq. (1), where one considers $\varphi_{r}, \partial_{\alpha_{1}} \varphi_{r}, \partial_{\alpha_{1}} \partial_{\alpha_{2}} \varphi_{r}, \cdots, \partial_{\alpha_{1}} \cdots \partial_{\alpha_{n}} \varphi_{r}$ as independent generalized coordinates. The Euler-Lagrange equa- tions are obtained from principle of least action

$$
\delta S=0
$$

where $\delta S$ is given by

$$
\delta S=\int d^{4} x \delta \mathcal{L}\left(\varphi_{r}, \partial_{\alpha_{1}} \varphi_{r}, \partial_{\alpha_{1} \alpha_{2}} \varphi_{r}, \cdots, \partial_{\alpha_{1} \cdots \alpha_{n}} \varphi_{r}\right)
$$

and is obtained by the variation of the generalized coordinates

$$
\begin{aligned}
\varphi_{r} & \longrightarrow \varphi_{r}+\delta \varphi_{r}, \\
\partial_{\alpha_{1}} \varphi_{r} & \longrightarrow \partial_{\alpha_{1}} \varphi_{r}+\delta \partial_{\alpha_{1}} \varphi_{r}, \\
\partial_{\alpha_{1} \alpha_{2}} \varphi_{r} & \longrightarrow \partial_{\alpha_{1} \alpha_{2}} \varphi_{r}+\delta \partial_{\alpha_{1} \alpha_{2}} \varphi_{r}, \\
, & \cdots, \\
\partial_{\alpha_{1} \cdots \alpha_{n}} \varphi_{r} & \longrightarrow \partial_{\alpha_{1} \cdots \alpha_{n}} \varphi_{r}+\delta \partial_{\alpha_{1} \cdots \alpha_{n}} \varphi_{r},
\end{aligned}
$$

with vanishing contributions on the surface of the integration volume as the boundary condition. The variation of the Lagrangian density with respect to all degrees of freedom reads

$$
\begin{aligned}
\delta \mathcal{L}= & \frac{\partial \mathcal{L}}{\partial \varphi_{r}} \delta \varphi_{r}+\frac{\partial \mathcal{L}}{\partial\left(\partial_{\alpha_{1}} \varphi_{r}\right)} \partial_{\alpha_{1}} \delta \varphi_{r}+\frac{\partial \mathcal{L}}{\partial\left(\partial_{\alpha_{1} \alpha_{2}} \varphi_{r}\right)} \partial_{\alpha_{1} \alpha_{2}} \delta \varphi_{r} \\
& \left.+\cdots+\frac{\partial \mathcal{L}}{\partial\left(\partial_{\alpha_{1} \cdots \alpha_{n}} \varphi_{r}\right)} \partial_{\alpha_{1} \cdots \alpha_{n}} \delta \varphi_{r}\right]
\end{aligned}
$$

As a next step one inserts Eq. (5) into Eq. (3) and then performs successively partial integrations, e.g., one partial integration for the second term in Eq. (5), two partial integrations for the third term in Eq. (5), and $n$ partial integrations for the last term. This procedure results in to the following integrand in Eq. (3)

$$
\begin{aligned}
\delta \mathcal{L}= & {\left[\frac{\partial \mathcal{L}}{\partial \varphi_{r}}-\partial_{\alpha_{1}} \frac{\partial \mathcal{L}}{\partial\left(\partial_{\alpha_{1}} \varphi_{r}\right)}+\partial_{\alpha_{1} \alpha_{2}} \frac{\partial \mathcal{L}}{\partial\left(\partial_{\alpha_{1} \alpha_{2}} \varphi_{r}\right)}\right.} \\
& \left.+\cdots+(-)^{n} \partial_{\alpha_{1} \cdots \alpha_{n}} \frac{\partial \mathcal{L}}{\partial\left(\partial_{\alpha_{1} \cdots \alpha_{n}} \varphi_{r}\right)}\right] \delta \varphi_{r}
\end{aligned}
$$

up to 4-divergence terms, which by Gauss law do not contribute to the action in Eq. (3). Thus, one arrives to the following generalized Euler-Lagrange equation

$$
\frac{\partial \mathcal{L}}{\partial \varphi_{r}}+\sum_{i=1}^{n}(-)^{i} \partial_{\alpha_{1} \cdots \alpha_{i}} \frac{\partial \mathcal{L}}{\partial\left(\partial_{\alpha_{1} \cdots \alpha_{i}} \varphi_{r}\right)}=0
$$

The Noether theorem follows from invariance principles of the Lagrangian density, Eq. (1), with respect to infinitesimal variations of the generalized coordinates and their argument $x^{\mu}$ (see for notations Appendix A). As further shown in Appendix $\mathrm{B}$, the requirement of invariance of the Lagrangian density, Eq. (1), with respect to global phase transformations

$$
\varphi_{r}(x) \longrightarrow \varphi_{r}^{\prime}(x)=e^{-i \epsilon} \varphi_{r}(x)
$$

leads to a continuity equation $\partial_{\mu} J^{\mu}=0$ for a conserved Noether current $J^{\mu}$. The latter is given by the following expression 


$$
J^{\mu}=-i\left[\mathcal{K}_{r}^{\mu} \varphi_{r}+\mathcal{K}_{r}^{\mu \sigma_{1}} \partial_{\sigma_{1}} \varphi_{r}+\mathcal{K}_{r}^{\mu \sigma_{1} \sigma_{2}} \partial_{\sigma_{1} \sigma_{2}} \varphi_{r}+\cdots+\mathcal{K}_{r}^{\mu \sigma_{1} \cdots \sigma_{n}} \partial_{\sigma_{1} \cdots \sigma_{n}} \varphi_{r}\right]
$$

In fact, for $n \rightarrow \infty$ the Noether current consists of an infinite sequence of tensors with increasing rank order. Furthermore, each of the different tensors $\mathcal{K}_{r}^{\mu \sigma_{1} \sigma_{2} \cdots}$ in Eq. (9) contains again infinite series terms of higher-order derivatives with respect to the Lagrangian density. They are given by the following expressions

$$
\begin{aligned}
\mathcal{K}_{r}^{\mu} & =\sum_{i=1}^{n}(-)^{i+1} \prod_{j=1}^{i-1} \partial_{\alpha_{j}} \frac{\partial \mathcal{L}}{\partial\left(\partial_{\mu \alpha_{j}} \varphi_{r}\right)}, \\
\mathcal{K}_{r}^{\mu \sigma_{1}} & =\sum_{i=1}^{n}(-)^{i+1} \prod_{j=1}^{i-1} \partial_{\alpha_{j}} \frac{\partial \mathcal{L}}{\partial\left(\partial_{\left.\mu \alpha_{j} \sigma_{1} \varphi_{r}\right)}\right.}, \\
\mathcal{K}_{r}^{\mu \sigma_{1} \sigma_{2}} & =\sum_{i=1}^{n}(-)^{i+1} \prod_{j=1}^{i-1} \partial_{\alpha_{j}} \frac{\partial \mathcal{L}}{\partial\left(\partial_{\left.\mu \alpha_{j} \sigma_{1} \sigma_{2} \varphi_{r}\right)}\right)} \\
\vdots & \\
\mathcal{K}_{r}^{\mu \sigma_{1} \cdots \sigma_{n}} & =\sum_{i=1}^{n}(-)^{i+1} \prod_{j=1}^{i-1} \partial_{\alpha_{j}} \frac{\partial \mathcal{L}}{\partial\left(\partial_{\mu \alpha_{j} \sigma_{1} \cdots \sigma_{n}} \varphi_{r}\right)} .
\end{aligned}
$$

The derivation of the energy-momentum tensor proceeds in a similar way, see Appendix B. Now the field arguments are transformed, but not the fields them self. In particular, invariance of the Lagrangian density (1) with respect to a constant displacement $\delta_{\mu}$ of the coordinates $x_{\mu}$

$$
x_{\mu} \longrightarrow x_{\mu}^{\prime}=x_{\mu}+\delta_{\mu},
$$

implies a continuity equation $\partial_{\mu} T^{\mu \nu}=0$ for the energy-momentum tensor $T^{\mu \nu}$ which takes the following form

$$
T^{\mu \nu}=\mathcal{K}_{r}^{\mu} \partial^{\nu} \varphi_{r}+\mathcal{K}_{r}^{\mu \sigma_{1}} \partial_{\sigma_{1}}^{\nu} \varphi_{r}+\mathcal{K}_{r}^{\mu \sigma_{1} \sigma_{2}} \partial_{\sigma_{1} \sigma_{2}}^{\nu} \varphi_{r}+\cdots+\mathcal{K}_{r}^{\mu \sigma_{1} \cdots \sigma_{n}} \partial_{\sigma_{1} \cdots \sigma_{n}}^{\nu} \varphi_{r}-g^{\mu \nu} \mathcal{L} .
$$

The 00-component of the energy-momentum tensor describes the energy density and the spatial diagonal components are related to the pressure density. These equations form a background for the construction and application of the NLD formalism presented in the proceeding sections. They will further provide a thermodynamically consistent framework for the calculation of the EoS in mean field approximation in terms of energy and pressure densities.

\section{THE NON-LINEAR DERIVATIVE MODEL}

In this section we introduce the non-linear derivative (NLD) model and derive the equations of motion for the relevant degrees of freedom. The NLD approach is essentially based on the Lagrangian density of RHD [2-4], which is given by

$$
\begin{aligned}
\mathcal{L} & =\frac{1}{2}\left[\bar{\Psi} \gamma_{\mu} i \vec{\partial}^{\mu} \Psi-\bar{\Psi} i \overleftarrow{\partial}^{\mu} \gamma_{\mu} \Psi\right]-m \bar{\Psi} \Psi-\frac{1}{2} m_{\sigma}^{2} \sigma^{2}+\frac{1}{2} \partial_{\mu} \sigma \partial^{\mu} \sigma-U(\sigma) \\
& +\frac{1}{2} m_{\omega}^{2} \omega_{\mu} \omega^{\mu}-\frac{1}{4} F_{\mu \nu} F^{\mu \nu}+\frac{1}{2} m_{\rho}^{2} \vec{\rho}_{\mu} \vec{\rho}^{\mu}-\frac{1}{4} \vec{G}_{\mu \nu} \vec{G}^{\mu \nu}-\frac{1}{2} m_{\delta}^{2} \vec{\delta}^{2}+\frac{1}{2} \partial_{\mu} \vec{\delta} \partial^{\mu} \vec{\delta}+\mathcal{L}_{i n t}
\end{aligned}
$$

where $\Psi=\left(\Psi_{p}, \Psi_{n}\right)^{T}$ denotes the nucleon spinor field in the Lagrangian density of a Dirac-type. In a spirit of RHD, the interactions between the nucleon fields are described by the exchange of meson fields. These are the scalar $\sigma$ and vector $\omega^{\mu}$ mesons in the isoscalar channel, as well as the scalar $\vec{\delta}$ and vector $\vec{\rho}^{\mu}$ mesons in the isovector channel. Their corresponding Lagrangian densities are of the Klein-Gordon and Proca types, respectively. The term $U(\sigma)=\frac{1}{3} b \sigma^{3}+\frac{1}{4} c \sigma^{4}$ contains the usual selfinteractions of the $\sigma$ meson. The notations for the masses of fields in Eq. (13) are obvious. The field strength tensors are defined as $F^{\mu \nu}=\partial^{\mu} \omega^{\nu}-\partial^{\nu} \omega^{\mu}$, $\vec{G}^{\mu \nu}=\partial^{\mu} \vec{\rho}^{\nu}-\partial^{\nu} \vec{\rho}^{\mu}$ for the isoscalar and isovector fields, respectively.

In conventional RHD approaches the interaction La- 
grangian $\mathcal{L}_{\text {int }}$ is given by [3, 4]

$$
\mathcal{L}_{\text {int }}=\mathcal{L}_{\text {int }}^{\sigma}+\mathcal{L}_{\text {int }}^{\omega}+\mathcal{L}_{\text {int }}^{\rho}+\mathcal{L}_{\text {int }}^{\delta},
$$

where

$$
\begin{gathered}
\mathcal{L}_{\text {int }}^{\sigma}=g_{\sigma} \bar{\Psi} \Psi \sigma, \\
\mathcal{L}_{\text {int }}^{\omega}=-g_{\omega} \bar{\Psi} \gamma^{\mu} \Psi \omega_{\mu}, \\
\mathcal{L}_{\text {int }}^{\rho}=-g_{\rho} \bar{\Psi} \gamma^{\mu} \vec{\tau} \Psi \vec{\rho}_{\mu}, \\
\mathcal{L}_{\text {int }}^{\delta}=g_{\delta} \bar{\Psi} \vec{\tau} \Psi \vec{\delta},
\end{gathered}
$$

and $\mathcal{L}_{i n t}^{\sigma, \omega, \rho, \delta}$ contains the meson-nucleon interactions with coupling strengths $g_{\sigma, \omega, \rho, \delta}$ and $\tau$ denotes the isospin Pauli operator.

In the NLD model the momentum dependence of fields is realized by the introduction of non-linear derivative operators in the interaction Lagrangian of conventional RHD. These additional operators regulate the high momentum components of the RMF fields in the interaction vertices and can be interpreted as cut-off form factors. This is in spirit of bosonexchange models where the phenomenological cut-off is an indispensable part of any microscopic description of mesonnucleon interaction [28, 29]. In the RMF (Hartree) approximation to RHD only bare Lorentz structures corresponding to the point-like meson-nucleon interactions are taken into account and the high momentum components of fields are not suppressed due to the missing nucleon finite size effect. The NLD model attempts to account for the suppression of the high momentum part of the nucleon field in the mesonnucleon interaction on a field-theoretical level.

The NLD interaction Lagrangians contain the conventional meson-nucleon RHD structures, however, they are extended by the inclusion of non-linear derivative operators into the meson-nucleon vertices. The NLD interaction Lagrangians followed here read

$$
\mathcal{L}_{\text {int }}^{\sigma}=\frac{g_{\sigma}}{2}[\bar{\Psi} \overleftarrow{\mathcal{D}} \Psi \sigma+\sigma \bar{\Psi} \overrightarrow{\mathcal{D}} \Psi]
$$

$$
\begin{gathered}
\mathcal{L}_{i n t}^{\omega}=-\frac{g_{\omega}}{2}\left[\bar{\Psi} \overleftarrow{\mathcal{D}} \gamma^{\mu} \Psi \omega_{\mu}+\omega_{\mu} \bar{\Psi} \gamma^{\mu} \overrightarrow{\mathcal{D}} \Psi\right] \\
\mathcal{L}_{\text {int }}^{\rho}=-\frac{g_{\rho}}{2}\left[\bar{\Psi} \overleftarrow{\mathcal{D}} \gamma^{\mu} \vec{\tau} \Psi \vec{\rho}_{\mu}+\vec{\rho}_{\mu} \bar{\Psi} \vec{\tau} \gamma^{\mu} \overrightarrow{\mathcal{D}} \Psi\right] \\
\mathcal{L}_{i n t}^{\delta}=\frac{g_{\delta}}{2}[\bar{\Psi} \overleftarrow{\mathcal{D}} \vec{\tau} \Psi \vec{\delta}+\vec{\delta} \bar{\Psi} \vec{\tau} \overrightarrow{\mathcal{D}} \Psi]
\end{gathered}
$$

As one can see, the only difference with respect to the conventional RHD interaction Lagrangian is the presence of additional operators $\overrightarrow{\mathcal{D}}, \overleftarrow{\mathcal{D}}$ which serve to regulate the high momentum component of the nucleon field. The hermiticity of the Lagrangian demands $\overleftarrow{\mathcal{D}}=\overrightarrow{\mathcal{D}}^{\dagger}$. The operator functions (regulators) $\overrightarrow{\mathcal{D}}, \overleftarrow{\mathcal{D}}$ are assumed to be generic functions of partial derivative operator and supposed to act on the nucleon spinors $\Psi$ and $\bar{\Psi}$, respectively. Furthermore, these regulators are assumed to be smooth functions. Therefore, the formal Taylor expansion of the operator functions in terms of partial derivatives generates an infinite series of higher-order derivative terms

$$
\begin{aligned}
& \overrightarrow{\mathcal{D}}:=\mathcal{D}(\vec{\xi})=\left.\sum_{j=0}^{n \rightarrow \infty} \frac{\partial^{j}}{\partial \vec{\xi} j} \mathcal{D}\right|_{\vec{\xi} \rightarrow 0} \frac{\vec{\xi}^{j}}{j !} \\
& \overleftarrow{\mathcal{D}}:=\mathcal{D}(\overleftarrow{\xi})=\left.\sum_{j=0}^{n \rightarrow \infty} \frac{\overleftarrow{\xi} j}{j !} \frac{\partial^{j}}{\partial \vec{\xi} j} \mathcal{D}\right|_{\overleftarrow{\xi} \rightarrow 0}
\end{aligned}
$$

The expansion coefficients are given by the partial derivatives of $\mathcal{D}$ with respect to the operator arguments $\vec{\xi}$ and $\overleftarrow{\xi}$ around the origin. The operators are defined as $\vec{\xi}=-\zeta^{\alpha} i \vec{\partial}_{\alpha}, \overleftarrow{\xi}=$ $i \overleftarrow{\partial}_{\alpha} \zeta^{\alpha}$ where the four vector $\zeta^{\mu}=v^{\mu} / \Lambda$ contains the cutoff $\Lambda$ and $v^{\mu}$ is an auxiliary vector. The functional form of the regulators is constructed such that in the limit $\Lambda \rightarrow \infty$ the following limit holds $\overrightarrow{\mathcal{D}}(\overleftarrow{\mathcal{D}}) \rightarrow 1$. Therefore, in the limit $\Lambda \rightarrow \infty$ the original RHD Lagrangians are recovered.

In the most general case the NLD formalism can be extended to the case of multiple variable regulators. In particular, we can assume the non-linear operator to be a multivariable non-linear function of higher-order partial derivatives, which are given by the following Taylor expansion

$$
\begin{aligned}
& \overrightarrow{\mathcal{D}}:=\mathcal{D}\left(\vec{\xi}_{1}, \vec{\xi}_{2}, \vec{\xi}_{3}, \vec{\xi}_{4}\right)=\sum_{i_{1}=0}^{n \rightarrow \infty} \sum_{i_{2}=0}^{n \rightarrow \infty} \sum_{i_{3}=0}^{n \rightarrow \infty} \sum_{i_{4}=0}^{n \rightarrow \infty} \frac{\partial^{i_{1}+i_{2}+i_{3}+i_{4}}}{\partial \vec{\xi}_{1}^{i_{1}} \partial \vec{\xi}_{2}^{i_{2}} \partial \vec{\xi}_{3}^{i_{3}} \partial \vec{\xi}_{4}^{i}} \mathcal{D}_{\left\{\vec{\xi}_{1}, \vec{\xi}_{2}, \vec{\xi}_{3}, \vec{\xi}_{4}\right\} \rightarrow 0} \frac{\vec{\xi}_{1}^{i_{1}} \vec{\xi}_{2}^{i_{2}} \vec{\xi}_{3}^{i_{3}} \vec{\xi}_{4}^{i_{4}}}{i_{1} ! i_{2} ! i_{3} ! i_{4} !}, \\
& \overleftarrow{\mathcal{D}}:=\mathcal{D}\left(\overleftarrow{\xi}_{1}, \overleftarrow{\xi}_{2}, \overleftarrow{\xi}_{3}, \overleftarrow{\xi}_{4}\right)=\left.\sum_{i_{1}=0}^{n \rightarrow \infty} \sum_{i_{2}=0}^{n \rightarrow \infty} \sum_{i_{3}=0}^{n \rightarrow \infty} \sum_{i_{4}=0}^{n \rightarrow \infty} \frac{\overleftarrow{\xi}_{1}^{i_{1}} \overleftarrow{\xi}_{2}^{i_{2}} \overleftarrow{\xi}_{3}^{i_{3}} \overleftarrow{\xi}_{4}^{i_{4}}}{i_{1} ! i_{2} ! i_{3} ! i_{4} !} \frac{\partial^{i_{1}+i_{2}+i_{3}+i_{4}}}{\partial \overleftarrow{\xi}_{1}^{i_{1}} \partial \overleftarrow{\xi}_{2}^{i_{2}} \partial \overleftarrow{\xi}_{3}^{i_{3}} \partial \overleftarrow{\xi}_{4}^{i_{4}}} \mathcal{D}\right|_{\left\{\overleftarrow{\xi}_{1}, \overleftarrow{\xi}_{2}, \overleftarrow{\xi}_{3}, \overleftarrow{\xi}_{4}\right\} \rightarrow 0}
\end{aligned}
$$

Then Eqs. (25) and (26) can be rearranged into the terms with increasing order with respect to the partial derivatives, see for details Appendix C. The operators $\xi_{i}$ are defined in a similar way as before

$$
\vec{\xi}_{i}=-\zeta_{i}^{\alpha} i \vec{\partial}_{\alpha}, \overleftarrow{\xi}_{i}=i \overleftarrow{\partial}_{\alpha} \zeta_{i}^{\alpha}
$$


with $\zeta_{i}^{\mu}=v_{i}^{\mu} / \Lambda(i=1,2,3,4)$ in this case. As we will show latter on, this representation allows to generate any desired form of the regulator function, i.e., momentum and/or energy dependent monopole, dipole etc. functions.

The derivation of the equation of motion for the Dirac field follows the generalized Euler-Lagrange equations, Eq. (7), to the NLD-Lagrangian density using the Taylor form of the regulators. This obviously will generate an infinite number of partial derivative terms in the equations of motions. However, as shown in detail in Appendix D these infinite series can be resummed (up to terms containing the derivatives of the meson fields) to the following Dirac equation

$$
\left[\gamma_{\mu}\left(i \partial^{\mu}-\Sigma^{\mu}\right)-\left(m-\Sigma_{s}\right)\right] \Psi=0,
$$

where the selfenergies $\Sigma^{\mu}$ and $\Sigma_{s}$ are given by

$$
\begin{aligned}
& \Sigma^{\mu}=g_{\omega} \omega^{\mu} \overrightarrow{\mathcal{D}}+g_{\rho} \vec{\tau} \cdot \vec{\rho}^{\mu} \overrightarrow{\mathcal{D}}+\cdots, \\
& \Sigma_{s}=g_{\sigma} \sigma \overrightarrow{\mathcal{D}}+g_{\delta} \vec{\tau} \cdot \vec{\delta} \overrightarrow{\mathcal{D}}+\cdots .
\end{aligned}
$$

Here both Lorentz-components of the selfenergy, $\Sigma^{\mu}$ and $\Sigma_{s}$, show an explicit linear behavior with respect to the meson fields $\sigma, \omega^{\mu}, \vec{\rho}^{\mu}$ and $\vec{\delta}$ as in the standard RMF. However, they contain an additional dependence on regulator functions.

The additional terms in Eqs. (29) and (30) containing the meson field derivatives are denoted by multiple dots. All these contributions can be also resummed. However, in the meanfield approximation to infinite nuclear matter, which will be discussed in the next section, these terms vanish. On the other hand, they will be needed in the description of finite systems, such as finite nuclei and heavy-ion collisions. Therefore, for simplicity we do not consider these terms here, and postpone the effect of these terms for future studies.

The derivation of the meson field equations of motion is straightforward, since here one has to use the standard EulerLagrange equations

$$
\frac{\partial \mathcal{L}}{\partial \varphi_{r}}-\partial_{\alpha} \frac{\partial \mathcal{L}}{\partial\left(\partial_{\alpha} \varphi_{r}\right)}=0
$$

where now $r=\sigma, \omega, \rho$ and $\delta$. The following Proca and KleinGordon equations are obtained

$$
\begin{aligned}
& \partial_{\alpha} \partial^{\alpha} \sigma+m_{\sigma}^{2} \sigma+\frac{\partial U}{\partial \sigma}=\frac{1}{2} g_{\sigma}[\bar{\Psi} \overleftarrow{\mathcal{D}} \Psi+\bar{\Psi} \overrightarrow{\mathcal{D}} \Psi] \\
& \partial_{\mu} F^{\mu \nu}+m_{\omega}^{2} \omega^{\nu}=\frac{1}{2} g_{\omega}\left[\bar{\Psi} \overleftarrow{\mathcal{D}} \gamma^{\nu} \Psi+\bar{\Psi} \gamma^{\nu} \overrightarrow{\mathcal{D}} \Psi\right] \\
& \partial_{\mu} \vec{G}^{\mu \nu}+m_{\rho}^{2} \vec{\rho}^{\nu}=\frac{1}{2} g_{\rho}\left[\bar{\Psi} \overleftarrow{\mathcal{D}} \gamma^{\nu} \vec{\tau} \Psi+\bar{\Psi} \vec{\tau} \gamma^{\nu} \overrightarrow{\mathcal{D}} \Psi\right] \\
& \partial_{\alpha} \partial^{\alpha} \vec{\delta}+m_{\sigma}^{2} \vec{\delta}=\frac{1}{2} g_{\delta}[\bar{\Psi} \overleftarrow{\mathcal{D}} \vec{\tau} \Psi+\bar{\Psi} \vec{\tau} \overrightarrow{\mathcal{D}} \Psi]
\end{aligned}
$$

Finally, we provide the general expressions for the Noether theorems within the NLD formalism. The evaluation of the conserved baryon current results from the application of the generalized expression for $J^{\mu}$, Eq. (9), to the Lagrangian density of the NLD model. As shown in detail in Appendix E, a systematic evaluation of the higher-order field derivatives of the NLD Lagrangian and the resummation procedure result in

$$
\begin{aligned}
J^{\mu}=\bar{\Psi} \gamma^{\mu} \Psi & -\frac{1}{2} g_{\sigma}\left[\bar{\Psi} \overleftarrow{\Omega}^{\mu} \Psi-\bar{\Psi} \vec{\Omega}^{\mu} \Psi\right] \sigma+\frac{1}{2} g_{\omega}\left[\bar{\Psi} \overleftarrow{\Omega}^{\mu} \gamma^{\alpha} \Psi-\bar{\Psi} \gamma^{\alpha} \vec{\Omega}^{\mu} \Psi\right] \omega_{\alpha} \\
& +\frac{1}{2} g_{\rho}\left[\bar{\Psi} \overleftarrow{\Omega}^{\mu} \gamma^{\alpha} \vec{\tau} \Psi-\bar{\Psi} \gamma^{\alpha} \vec{\Omega}^{\mu} \vec{\tau} \Psi\right] \vec{\rho}_{\alpha}-\frac{1}{2} g_{\delta}\left[\bar{\Psi} \overleftarrow{\Omega}^{\mu} \vec{\tau} \Psi-\bar{\Psi} \vec{\Omega}^{\mu} \vec{\tau} \Psi\right] \vec{\delta}+\cdots
\end{aligned}
$$

The new non-linear derivative operators in Eq. (36), $\overleftarrow{\Omega}^{\mu}:=$ $\partial \overleftarrow{\mathcal{D}} / \partial\left(i \overleftarrow{\partial}_{\mu}\right)$ and $\vec{\Omega}^{\mu}:=\partial \overrightarrow{\mathcal{D}} / \partial\left(i \vec{\partial}_{\mu}\right)$, denote the derivatives of $\overleftarrow{\mathcal{D}}$ and $\overrightarrow{\mathcal{D}}$ with respect to their operator argument $i \overleftarrow{\partial}_{\mu}$ and $i \vec{\partial}_{\mu}$ (see Appendix E). The first term in Eq. (36) corresponds to the standard expression of the RHD models and the additional contributions arise due to the additional higherorder field derivatives in the Noether theorem, Eq. (9).

The energy-momentum tensor, $T^{\mu \nu}$, is determined according to Eq. (12). The evaluation procedure, which is similar to that one for the Noether current, results in the following NLD expression for $T^{\mu \nu}$

$$
\begin{aligned}
T^{\mu \nu} & =\frac{1}{2} \bar{\Psi} \gamma^{\mu} i \vec{\partial}^{\nu} \Psi-\frac{1}{2} \bar{\Psi} i \overleftarrow{\partial}^{\nu} \gamma^{\mu} \Psi \\
& +\frac{1}{2} g_{\sigma}\left[\bar{\Psi} \vec{\Omega}^{\mu} i \vec{\partial}^{\nu} \Psi+\bar{\Psi} i \overleftarrow{\partial}^{\nu} \overleftarrow{\Omega}^{\mu} \Psi\right] \sigma-\frac{1}{2} g_{\omega}\left[\bar{\Psi} \gamma^{\alpha} \vec{\Omega}^{\mu} i \vec{\partial}^{\nu} \Psi+\bar{\Psi} i \overleftarrow{\partial}^{\nu} \overleftarrow{\Omega}^{\mu} \gamma^{\alpha} \Psi\right] \omega_{\alpha} \\
& -\frac{1}{2} g_{\rho}\left[\bar{\Psi} \vec{\tau} \gamma^{\alpha} \vec{\Omega}^{\mu} i \vec{\partial}^{\nu} \Psi+\bar{\Psi} i \overleftarrow{\partial}^{\nu} \overleftarrow{\Omega}^{\mu} \gamma^{\alpha} \vec{\tau} \Psi\right] \vec{\rho}_{\alpha}+\frac{1}{2} g_{\delta}\left[\bar{\Psi} \vec{\tau}^{\mu} i \vec{\partial}^{\nu} \Psi+\bar{\Psi} i \overleftarrow{\partial}^{\nu} \overleftarrow{\Omega}^{\mu} \vec{\tau} \Psi\right] \vec{\delta} \\
& -g^{\mu \nu} \mathcal{L}+\cdots
\end{aligned}
$$

The first line in Eq. (37) is just the usual kinetic RHD con-

tribution to $T^{\mu \nu}$, while the additional kinetic terms originate 
from the evaluation of the higher-order derivatives in Eq. (12). These terms will be important for the the thermodynamic consistency of the model and the validation of the HugenholtzVan Hove theorem [14, 30]. Again the terms not shown in Eqs. (36) and (37) describe the contribution of terms containing the derivatives of the meson fields.

\section{RMF APPROACH TO INFINITE NUCLEAR MATTER}

In the mean-field approximation the mesons are treated as classical fields. Infinite nuclear matter is described by a static homogeneous, isotropic, spin and isospin-saturated system of protons and neutrons. In this case, the spatial components of the Lorentz-vector meson fields vanish with $\omega^{\mu} \rightarrow\left(\omega^{0}, \overrightarrow{0}\right)$, and in isospin space only the neutral component of the isovector fields survive, i.e., $\vec{\rho}^{\mu} \rightarrow\left(\rho_{3}^{0}, \overrightarrow{0}\right)$ and $\vec{\delta} \rightarrow \delta_{3}$. For simplicity, we denote in the following the third isospin components of the isovector fields as $\rho$ and $\delta$.

The derivation of the RMF equations starts with the usual plane wave ansatz

$$
\Psi_{i}(s, \vec{p})=u_{i}(s, \vec{p}) e^{-i p^{\mu} x_{\mu}},
$$

where $i$ stands for protons $(i=p)$ or neutrons $(i=n)$ and $p^{\mu}=(E, \vec{p})$ is a single nucleon 4-momentum. The application of the non-linear derivative operator $\mathcal{D}$ to the plane wave ansatz of the spinor fields results in

$$
\begin{aligned}
& \mathcal{D}(\vec{\xi}) \Psi_{i}=\mathcal{D}(\xi) u_{i}(s, \vec{p}) e^{-i p^{\mu} x_{\mu}}, \\
& \bar{\Psi}_{i} \mathcal{D}(\overleftarrow{\xi})=\mathcal{D}(\xi) \bar{u}_{i}(s, \vec{p}) e^{+i p^{\mu} x_{\mu}},
\end{aligned}
$$

where the regulators in the r.h.s. of above equation are now functions of the scalar argument $\xi=-\frac{v_{\alpha} p^{\alpha}}{\Lambda}$.

With the help of Eqs. (38) and (39) one gets the Dirac equation similar to Eq. (28) with selfenergies given by

$$
\begin{gathered}
\Sigma_{v i}^{\mu}=g_{\omega} \omega^{\mu} \mathcal{D}+g_{\rho} \tau_{i} \rho^{\mu} \mathcal{D}, \\
\Sigma_{s i}=g_{\sigma} \sigma \mathcal{D}+g_{\delta} \tau_{i} \delta \mathcal{D},
\end{gathered}
$$

where now $\tau_{i}=+1$ for protons $(i=p)$ and $\tau_{i}=-1$ for neutrons $(i=n)$. We note again that in the RMF approximation to infinite matter the additional terms including the meson field derivatives vanish. This largely simplifies the formalism, since these terms which show up in the original Dirac equation, see Eq. (28), do not appear any more.

The solutions of the Dirac equation takes the form

$$
u_{i}(s, \vec{p})=N_{i}\left(\begin{array}{c}
\varphi_{s} \\
\frac{\vec{\sigma} \cdot \vec{p}}{E_{i}^{*}+m_{i}^{*}} \varphi_{s}
\end{array}\right)
$$

with spin eigenfunctions $\varphi_{s}$, the in-medium energy

$$
E_{i}^{*}:=E-\Sigma_{v i}^{0},
$$

and the Dirac mass

$$
m_{i}^{*}:=m-\Sigma_{s i}
$$

For a given momentum the single particle energy $E$ is obtained from the in-medium on-shell relation

$$
E_{i}^{* 2}-\vec{p}^{2}=m_{i}^{* 2}
$$

The factor $N_{i}$ is determined from the normalization of the probability distribution, that is $\int d^{3} x J^{0}=1$. In the conventional RMF models the baryon density is given by the familiar expression $J^{0}=\Psi^{\dagger} \Psi$ and the normalization condition $\int d^{3} x \Psi^{\dagger} \Psi=1$ would result in $N_{i}=\sqrt{\frac{E_{i}^{*}+m_{i}^{*}}{2 E_{i}^{*}}}$. In the NLD model one has to use Eq. (9) for the Noether current by keeping in mind that the infinite series of meson field derivatives vanish in the RMF approach to nuclear matter. In this case, see again Appendix E for details, the conserved baryon current $J^{\mu}$ is resummed up to infinity and the result reads

$$
\begin{aligned}
J^{\mu}= & \sum_{i=p, n}\left[\left\langle\bar{\Psi}_{i} \gamma^{\mu} \Psi_{i}\right\rangle\right. \\
& +g_{\sigma}\left\langle\bar{\Psi}_{i}\left[\partial_{p}^{\mu} \mathcal{D}\right] \Psi_{i}\right\rangle \sigma-g_{\omega}\left\langle\bar{\Psi}_{i}\left[\partial_{p}^{\mu} \mathcal{D}\right] \gamma^{\alpha} \Psi_{i}\right\rangle \omega_{\alpha} \\
& \left.-g_{\rho} \tau_{i}\left\langle\bar{\Psi}_{i}\left[\partial_{p}^{\mu} \mathcal{D}\right] \gamma^{\alpha} \Psi_{i}\right\rangle \rho_{\alpha}+g_{\delta} \tau_{i}\left\langle\bar{\Psi}_{i}\left[\partial_{p}^{\mu} \mathcal{D}\right] \Psi_{i}\right\rangle \delta\right]
\end{aligned}
$$

The 0-component of the Noether current describes the conserved nucleon density $\rho_{B}=J^{0}$, from which also the relation between the Fermi momentum $p_{F}$ and $\rho_{B}$ is uniquely determined. In particular, using the Gordon identity and Eqs. (41) and (42) for the RMF selfenergies, one obtains

$$
\begin{aligned}
J^{\mu} & =\frac{\kappa}{(2 \pi)^{3}} \sum_{i=p, n} \int_{|\vec{p}| \leq p_{F_{i}}} d^{3} p N_{i}^{2} \\
& \times\left[\frac{p_{i}^{* \mu}}{E_{i}^{*}}+\left(\partial_{p}^{\mu} \Sigma_{s i}\right) \frac{m_{i}^{*}}{E_{i}^{*}}-\left(\partial_{p}^{\mu} \Sigma_{v i}^{\beta}\right) \frac{p_{i \beta}^{*}}{E_{i}^{*}}\right],
\end{aligned}
$$

where $\kappa=2$ is a spin degeneracy factor, $p_{F_{i}}$ stands for the proton or neutron Fermi-momentum and the effective momentum is given by

$$
p_{i}^{* \mu}=p^{\mu}-\Sigma_{v i}^{\mu} .
$$

One defines now a new in-medium 4-momentum $\Pi_{i}^{\mu}$ as

$$
\Pi_{i}^{\mu}=p_{i}^{* \mu}+m_{i}^{*}\left(\partial_{p}^{\mu} \Sigma_{s i}\right)-\left(\partial_{p}^{\mu} \Sigma_{v i}^{\beta}\right) p_{i \beta}^{*},
$$

and arrives to the following expression

$$
J^{\mu}=\frac{\kappa}{(2 \pi)^{3}} \sum_{i=p, n} \int_{|\vec{p}| \leq p_{F_{i}}} d^{3} p N_{i}^{2} \frac{\Pi_{i}^{\mu}}{E_{i}^{*}} .
$$

On the other hand, the general definition of the baryon current results from the covariant superposition of all the occupied in-medium on-shell nucleon positive energy states up to the proton or neutron Fermi momentum [7]

$$
\begin{aligned}
J^{\mu} & =\frac{\kappa}{(2 \pi)^{3}} \sum_{i=p, n} \int_{|\vec{p}| \leq p_{F_{i}}} d^{4} p \\
& \times \Pi_{i}^{\mu} \delta\left(p_{i}^{* \mu} p_{i \mu}^{*}-m_{i}^{* 2}\right) 2 \Theta\left(p^{0}\right) .
\end{aligned}
$$


In the NLD approach the mean-field selfenergies depend explicitly on the single-particle momentum $p^{\mu}$. Therefore, using the properties of the $\delta$-function the time-like $d p^{0}$ component can be integrated out explicitly. The result reads

$$
J^{\mu}=\frac{\kappa}{(2 \pi)^{3}} \sum_{i=p, n} \int_{|\vec{p}| \leq p_{F_{i}}} d^{3} p \frac{\Pi_{i}^{\mu}}{\Pi_{i}^{0}} .
$$

Comparing Eq. (52) with the equation for the NLD current, Eq. (50), one gets the following result for the normalization

$$
N_{i}=\sqrt{\frac{E_{i}^{*}+m_{i}^{*}}{2 E_{i}^{*}}} \sqrt{\frac{E_{i}^{*}}{\Pi_{i}^{0}}}
$$

and the bilinear products between the in-medium spinors of protons and neutrons are given by

$$
\begin{aligned}
\bar{u}_{i}(p) u_{i}(p) & =\frac{m_{i}^{*}}{\Pi_{i}^{0}}, \\
\bar{u}_{i}(p) \gamma^{0} u_{i}(p) & =1
\end{aligned}
$$

Eq. (55) ensures also the proper normalization of the probability distribution, i.e., $\int \Psi^{\dagger} \Psi=1$.

In our first work [25], where the non-linear derivative model has been proposed, the correction terms proportional to the partial derivatives of the selfenergies with respect to the single-particle momentum $p^{\mu}$ in Eq. (49) were not taken into account. Even if their contributions are small at low densities, these terms will be included in the present calculations which attempt to consider also the high density domain of the EoS in neutron stars. On the other hand, the inclusion of these terms is crucial for a fully thermodynamically consistent formalism and is independent of the particular form of the cut-off functions. Note that, the additional cut-off dependent terms in the baryon and energy densities of Ref. [25] are now canceled by the proper normalization conditions.

The energy-momentum tensor in NLD is obtained by applying the Noether theorem for translational invariance. In nuclear matter the resummation procedure results in the following expression

$$
\begin{aligned}
T^{\mu \nu}= & \sum_{i=p, n}\left[\left\langle\bar{\Psi}_{i} \gamma^{\mu} p^{\nu} \Psi_{i}\right\rangle\right. \\
& \left.+g_{\sigma}\left\langle\bar{\Psi}_{i}\left[\partial_{p}^{\mu} \mathcal{D}\right] p^{\nu} \Psi_{i}\right\rangle \sigma-g_{\omega}\left\langle\bar{\Psi}_{i}\left[\partial_{p}^{\mu} \mathcal{D}\right] \gamma^{\alpha} p^{\nu} \Psi_{i}\right\rangle \omega_{\alpha}-g_{\rho} \tau_{i}\left\langle\bar{\Psi}_{i}\left[\partial_{p}^{\mu} \mathcal{D}\right] \gamma^{\alpha} p^{\nu} \Psi_{i}\right\rangle \rho_{\alpha}+g_{\delta} \tau_{i}\left\langle\bar{\Psi}_{i}\left[\partial_{p}^{\mu} \mathcal{D}\right] p^{\nu} \Psi_{i}\right\rangle \delta_{\alpha}\right] \\
& -g^{\mu \nu}\langle\mathcal{L}\rangle .
\end{aligned}
$$

The evaluation of the expectation values in Eq. (56) can be done in a similar way as for the current with the result

$$
T^{\mu \nu}=\sum_{i=p, n} \frac{\kappa}{(2 \pi)^{3}} \int_{|\vec{p}| \leq p_{F_{i}}} d^{3} p \frac{p^{\nu}}{\Pi_{i}^{0}}\left[p_{i}^{* \mu}+m_{i}^{*}\left(\partial_{p}^{\mu} \Sigma_{s i}\right)-p_{i}^{* \alpha}\left(\partial_{p}^{\mu} \Sigma_{\alpha i}\right)\right]-g^{\mu \nu}\langle\mathcal{L}\rangle .
$$

Using Eq. (49) one arrives to the final expression for the energy-momentum tensor in the NLD formalism, which can be written in the following form

$$
T^{\mu \nu}=\sum_{i=p, n} \frac{\kappa}{(2 \pi)^{3}} \int_{|\vec{p}| \leq p_{F_{i}}} d^{3} p \frac{\Pi_{i}^{\mu} p^{\nu}}{\Pi_{i}^{0}}-g^{\mu \nu}\langle\mathcal{L}\rangle,
$$

from which the energy density $\varepsilon \equiv T^{00}$ and the pressure $P$ can be calculated, i.e.,

$$
\begin{aligned}
& \varepsilon=\sum_{i=p, n} \frac{\kappa}{(2 \pi)^{3}} \int_{|\vec{p}| \leq p_{F_{i}}} d^{3} p E(\vec{p})-\langle\mathcal{L}\rangle, \\
& P=\frac{1}{3} \sum_{i=p, n} \frac{\kappa}{(2 \pi)^{3}} \int_{|\vec{p}| \leq p_{F_{i}}} d^{3} p \frac{\vec{\Pi}_{i} \cdot \vec{p}}{\Pi_{i}^{0}}+\langle\mathcal{L}\rangle .
\end{aligned}
$$

Eqs. (59) and (60) look similar as the familiar expressions of the usual RMF models. However, the non-linear effects in- duced by the regulators show up through the generalized momentum $\Pi_{i}^{\mu}$ and through the dispersion relation for the singleparticle energy $E(\vec{p})$. Note the different form of the generalized momentum, $\Pi_{i}^{\mu}$, when one chooses energy or momentum dependent cut-off functions. Indeed, in the latter case the spatial derivatives in $\vec{\Pi}_{i}$ contribute in the pressure, while for energy-dependent cut-off functions they vanish and $\vec{\Pi}_{i}=\vec{p}$ holds. In any case, the expressions for the energy-density and pressure within the conventional RMF models are recovered by simple replacement $\Pi_{i}^{\mu} \rightarrow p_{i}^{* \mu}$, which is just equivalent to taking the limiting case $\Lambda \rightarrow \infty$ in the NLD expressions.

Finally, the NLD meson-field equations in the RMF ap- 
proach to nuclear matter read

$$
\begin{aligned}
m_{\sigma}^{2} \sigma+\frac{\partial U}{\partial \sigma} & =g_{\sigma} \sum_{i=p, n}\left\langle\bar{\Psi}_{i} \mathcal{D} \Psi_{i}\right\rangle=g_{\sigma} \rho_{s}, \\
m_{\omega}^{2} \omega & =g_{\omega} \sum_{i=p, n}\left\langle\bar{\Psi}_{i} \gamma^{0} \mathcal{D} \Psi_{i}\right\rangle=g_{\omega} \rho_{0}, \\
m_{\rho}^{2} \rho & =g_{\rho} \sum_{i=p, n} \tau_{i}\left\langle\bar{\Psi}_{i} \gamma^{0} \mathcal{D} \Psi_{i}\right\rangle=g_{\rho} \rho_{I}, \\
m_{\delta}^{2} \delta & =g_{\delta} \sum_{i=p, n} \tau_{i}\left\langle\bar{\Psi}_{i} \mathcal{D} \Psi_{i}\right\rangle=g_{\delta} \rho_{I S}
\end{aligned}
$$

Using Eqs. (54) and (55), the evaluation of the source terms of the meson-field equations is straightforward. In particular, the scalar-isoscalar $\rho_{s}$, vector-isoscalar $\rho_{0}$, vector-isovector $\rho_{I}$ and scalar-isovector $\rho_{I S}$ are given

$$
\begin{gathered}
\rho_{s}=\frac{\kappa}{(2 \pi)^{3}} \sum_{i=p, n} \int_{|\vec{p}| \leq p_{F_{i}}} d^{3} p \frac{m_{i}^{*}}{\Pi_{i}^{0}} \mathcal{D}(p), \\
\rho_{0}=\frac{\kappa}{(2 \pi)^{3}} \sum_{i=p, n} \int_{|\vec{p}| \leq p_{F_{i}}} d^{3} p \frac{E_{i}^{*}}{\Pi_{i}^{0}} \mathcal{D}(p), \\
\rho_{I}=\rho_{0 p}-\rho_{0 n}, \\
\rho_{I S}=\rho_{s p}-\rho_{s n} .
\end{gathered}
$$

The meson-field equations of motion show a similar structure as those of the standard RMF approximation. For example, the scalar-isoscalar density $\rho_{s}$ is suppressed with respect to the vector density $\rho_{0}$ by the factor $m_{i}^{*} / \Pi_{i}^{0}$, in a similar way as in the conventional Walecka models [3]. However, the substantial difference between NLD and conventional RMF appears in the source terms which now contain in addition the momentum-dependent regulator $\mathcal{D}$.

\section{RESULTS}

\section{A. Model parameters}

The non-linear derivative operators, $\mathcal{D}$, in the NLD Lagrangian are not constrained from first principles and allows us to consider different functional forms of $\mathcal{D}$. In nuclear matter, these regulators can be chosen as functions of the singleparticle energy or momentum, depending on the choice of the auxiliary multi-dimensional parameters $\zeta_{i}^{\mu}$. The available constraints are the bulk properties of nuclear matter and the empirically known energy dependence of the in-medium optical potential. It is also well known $[9,31,32]$ that the selfenergies should decrease or saturate as function of baryon density and single-particle 4-momentum. In the NLD model all these features of the relativistic mean-fields can be realized using energy or momentum dependent form factors which regulate the high energy (momentum) behavior of the nucleon 4-momentum.
Various choices of the regulator functions are possible. We have done calculations for different forms of $\mathcal{D}$ and found that the simplest momentum dependent monopole form factor provides the best description of the low and high density nuclear matter properties and agrees very well with the empirical momentum dependence of the in-medium Schrödingerequivalent optical potential. A momentum-dependent cut-off of a monopole form can be obtained by the following choice

$$
\overrightarrow{\mathcal{D}}=\left[\frac{1}{1+\sum_{j=1}^{4}\left(\zeta_{j}^{\alpha} i \vec{\partial}_{\alpha}\right)^{2}}\right],
$$

with $v_{1}^{\alpha}=(0,0,0,0), v_{2}^{\alpha}=(0,1,0,0), v_{3}^{\alpha}=(0,0,1,0)$ and $v_{4}^{\alpha}=(0,0,0,1)$. In nuclear matter this results in

$$
\mathcal{D}=\frac{\Lambda^{2}}{\Lambda^{2}+\vec{p}^{2}}
$$

Furthermore, in our fit to bulk properties of nuclear matter we use different cut-off parameters $\Lambda_{s} \equiv \Lambda_{\sigma}$ and $\Lambda_{v} \equiv \Lambda_{\omega}=\Lambda_{\rho}$ for the scalar and vector meson-nucleon vertices, respectively, and we neglect in the following the contribution of the $\delta$ meson.

The NLD model contains in total eight parameters. These are the meson-nucleon couplings $g_{\sigma}, g_{\omega}$ and $g_{\rho}$, the parameters $b$ and $c$ of the selfinteractions of the $\sigma$-meson, the mass $m_{\sigma}$ of the $\sigma$-meson, and the cut-offs $\Lambda_{s}$ and $\Lambda_{v}$. In principle, the masses of the $\omega$ - and $\rho$-mesons should be also included in the fit. In all the calculations concerning the fit the results for these two masses turned out to be always around their free values. Therefore, we keep for $m_{\omega}$ and $m_{\rho}$ the bare masses. Seven parameters, i.e., $g_{\sigma}, g_{\omega}, b$ and $c, \Lambda_{s}, \Lambda_{v}$ and $m_{\sigma}$ are adjusted to the bulk properties of symmetric nuclear matter and to the empirical energy dependence of the in-medium optical potential. The remaining parameter $g_{\rho}$ is determined by the experimentally known symmetry energy value at saturation density. The constraints in symmetric nuclear matter are the binding energy per nucleon $E_{b} \equiv \varepsilon / \rho_{B}-m$ and the compression modulus $K=\left.9 \rho_{\text {sat }}^{2} \frac{\partial^{2} E_{b}}{\partial \rho_{B}^{2}}\right|_{\rho_{B}=\rho_{s a t}}$ at the ground-state or saturation density $\rho_{\text {sat }}$, and the saturation density $\rho_{\text {sat }}$ itself. Furthermore, the momentum dependence is fixed by the optical potential $U_{\text {opt }}$ at ground-state density and at two kinetic energies. First, at $E_{k i n}=205 \mathrm{MeV}$ (where $U_{\text {opt }}=0$ $\mathrm{MeV}$ ) and at $E_{\text {kin }}=1000 \mathrm{MeV}$.

The numerical calculations for the fit procedure have been performed using the Nelder-Mead minimization algorithm NELMIN [33]. Furthermore, an adaptive non-linear leastsquare package NL2SOL [34, 35] has been supplemented in order to test robustness of the fit procedure. The experimental data used in the fit are supplemented by the corresponding errors as provided by the empirical information in Table I. At each iteration step of the minimization routines, the coupled set of the NLD equations has been solved with a Powellhybrid method as provided by the HYBRD routine [36]. The integrals which appear in the source terms of the mesonfield equations have been treated numerically using an adaptive Gauss algorithm. Note that, in the NLD model with the 


\begin{tabular}{|c|c|c|c|c|c|c|c|c|}
\hline Model & $\rho_{\text {sat }}$ & $E_{b}$ & $K$ & $a_{\text {sym }}$ & $L$ & $K_{\text {sym }}$ & $K_{a s y}$ & Ref. \\
\hline & {$\left[\mathrm{fm}^{-3}\right]$} & {$[\mathrm{MeV} / A]$} & {$[\mathrm{MeV}]$} & {$[\mathrm{MeV}]$} & {$[\mathrm{MeV}]$} & {$[\mathrm{MeV}]$} & {$[\mathrm{MeV}]$} & \\
\hline NLD & 0.156 & -15.30 & 251 & 30 & 81 & -28 & -514 & this work \\
\hline NL3* & 0.150 & -16.31 & 258 & 38.68 & 125.7 & 104.08 & -650.12 & [37] \\
\hline DD & 0.149 & -16.02 & 240 & 31.60 & 56 & -95.30 & -431.30 & [19] \\
\hline $\mathrm{D}^{3} \mathrm{C}$ & 0.151 & -15.98 & 232.5 & 31.90 & 59.30 & -74.7 & -430.50 & [22] \\
\hline \multirow[t]{2}{*}{ DBHF } & 0.185 & -15.60 & 290 & 33.35 & 71.10 & -27.1 & -453.70 & {$[38,39]$} \\
\hline & 0.181 & -16.15 & 230 & 34.20 & 71 & 87.36 & -340 & [40] \\
\hline empirical & $\begin{array}{c}0.167 \pm 0.019 \\
{[41-43]}\end{array}$ & $\begin{array}{c}-16 \pm 1 \\
{[41-43]}\end{array}$ & $\begin{array}{c}230 \pm 10 \\
{[43-45]}\end{array}$ & $\begin{array}{c}31.1 \pm 1.9 \\
{[46]}\end{array}$ & $\begin{array}{c}88 \pm 25 \\
{[46]}\end{array}$ & $\begin{array}{l}- \\
-\end{array}$ & $\begin{array}{c}-550 \pm 100 \\
{[47]}\end{array}$ & \\
\hline
\end{tabular}

TABLE I. Bulk saturation properties of nuclear matter, i.e., saturation density $\rho_{\text {sat }}$, binding energy per nucleon $E_{b}$, compression modulus $K$, asymmetry parameter $a_{\text {sym }}$, slope and curvature parameters $L$ and $K_{s y m}$, respectively, and the observable $K_{a s y}$ in the NLD model. Our results are compared with the non-linear Walecka parametrization NL3* , the density dependent DD and the derivative coupling $\mathrm{D}^{3} \mathrm{C}$ models as well as with two versions of the microscopic DBHF approach. The empirical values are shown too.

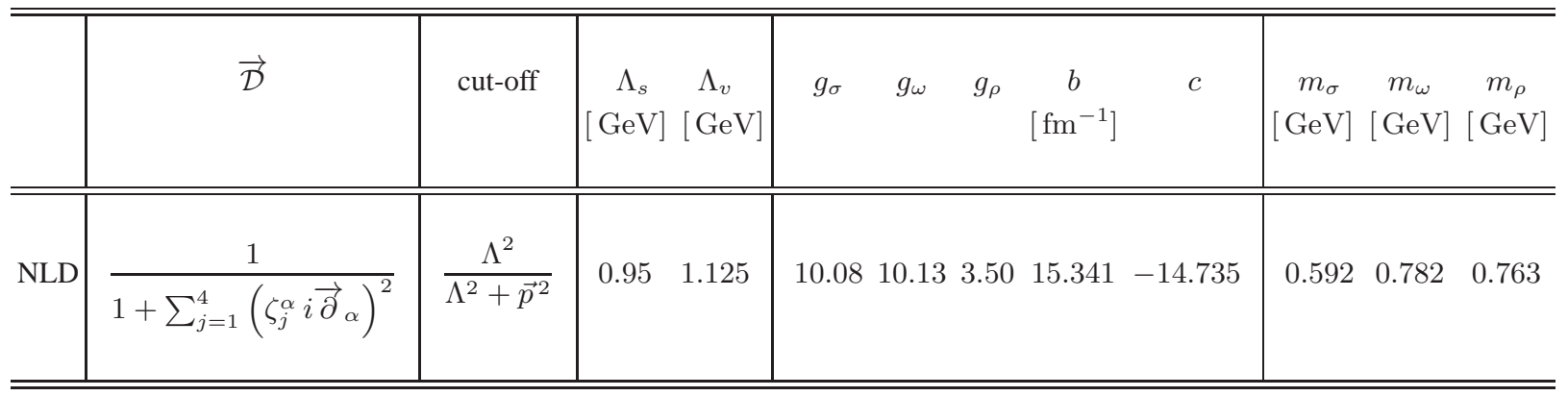

TABLE II. The parameters of the NLD model. First and second columns show the form of the non-linear operator and the regulator in nuclear matter, respectively. The other columns show the values of the parameters, as resulted from the fit to nuclear matter bulk properties.

momentum-dependent regulator functions the solution of the dispersion relation for the single particle energy $E$, Eq. (46), does not involve additional complex root-finding algorithms, because the RMF selfenergies depend on the nucleon momentum $p$ only, and not on the particle energy as in our previous works [23-25].

The functional form of the non-linear operator, its regulator (cut-off) in nuclear matter and the fit parameters of the NLD model are shown in Table II. Table I shows the extracted bulk properties of nuclear matter, i.e., the binding energy, the compression modulus, the asymmetry parameter $a_{\text {sym }}=E_{\text {sym }}\left(\rho_{\text {sat }}\right)$ ( $E_{\text {sym }}$ is the symmetry energy), the slope and curvature parameters, $L=\left.3 \rho_{\text {sat }} \frac{\partial E_{s y m}}{\partial \rho_{B}}\right|_{\rho_{B}=\rho_{\text {sat }}}$ and $K_{a s y}=K_{\text {sym }}-6 L$ (with $K_{\text {sym }}=\left.9 \rho_{\text {sat }}^{2} \frac{\partial^{2} E_{s y m}}{\partial \rho_{B}^{2}}\right|_{\rho_{B}=\rho_{s a t}}$ being the compressibility of the symmetry energy), respectively, in the NLD model, and in comparison with other RMF models widely used in the literature. These are the NL3* parametrization [37], the density dependent (DD) and derivative coupling $\left(\mathrm{D}^{3} \mathrm{C}\right)$ models $[19,22]$. The bulk nuclear matter properties in the NLD model are comparable with those re- 


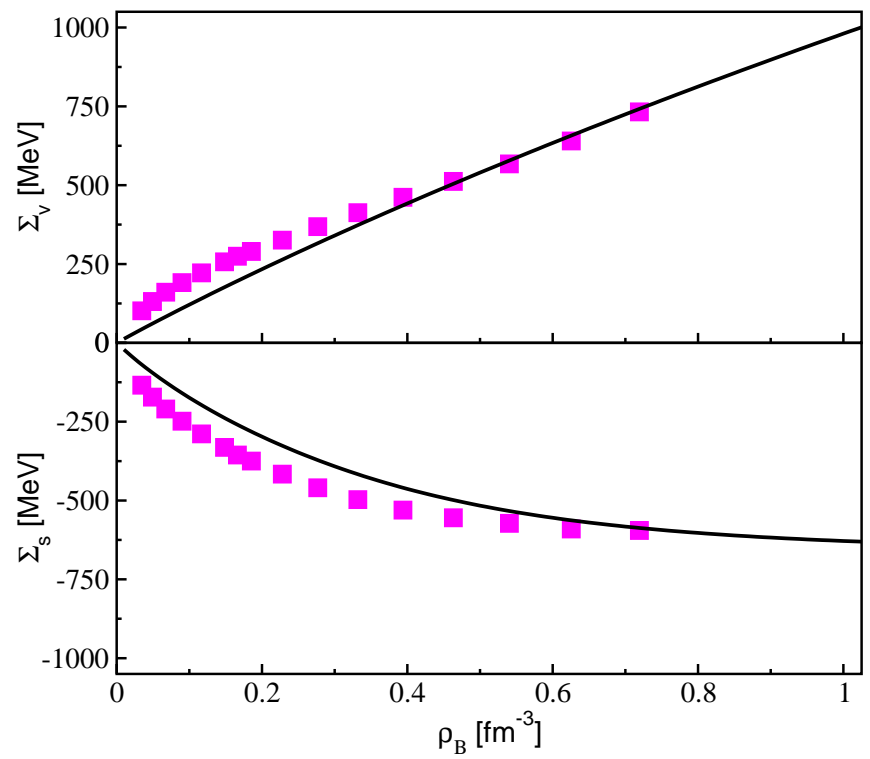

FIG. 1. Vector (upper panel) and scalar (lower panel) selfenergies as function of the baryon density $\rho_{B}$ in the NLD model (solid curves) and in the DBHF approach (filled squares) [39]. The calculations refer to isospin-symmetric $(\alpha=0)$ nuclear matter.

sults in the NL3*, DD and $\mathrm{D}^{3} \mathrm{C}$ parameterizations, while for the saturation density and the slope and curvature parameters of the symmetry energy the NLD calculation is closer to the empirical data. It is interesting that the NLD results are also comparable and in some cases even better than the DBHF calculations.

As we will discuss later on in more details, the NLD model with the same parameters of Table II describes remarkably well the empirical energy dependence of the Schrödingerequivalent optical potential. This is not the case in standard RMF approaches, such as the NL3* and DD models, except if one uses supplementary derivative interactions $\left(D^{3} C\right)$, however, with the cost of many additional parameters [22].

\section{B. The NLD equation of state}

We start the discussion of the NLD calculations with the density dependence of the relativistic mean-fields. Fig. 1 shows the density dependence of the nucleon selfenergies for isospin-symmetric nuclear matter in the NLD model. Our results are compared also with DBHF calculations, widely used in the literature [39]. It is remarkable that a simple folding of the meson-nucleon couplings $g_{i}(i=\sigma, \omega, \rho)$ with the cut-off function $\mathcal{D}$ results in a very similar density dependence of the of NLD selfenergies as compared to the DBHF selfenergies, in particular, for densities above $\rho_{B}>0.3-0.4 \mathrm{fm}^{-3}$. Note again that for momentum dependent cut-off functions the standard normalization of the spinors, i.e., $N_{i}=\sqrt{\frac{E_{i}^{*}+m_{i}^{*}}{2 E_{i}^{*}}}$, is not affected in this case, since $\Pi_{i}^{0}=E_{i}^{*}$ holds, and according to Eq. (49) only the pressure is modified, as shown in Eq. (60).

Fig. 2 shows the equation of state in terms of the binding

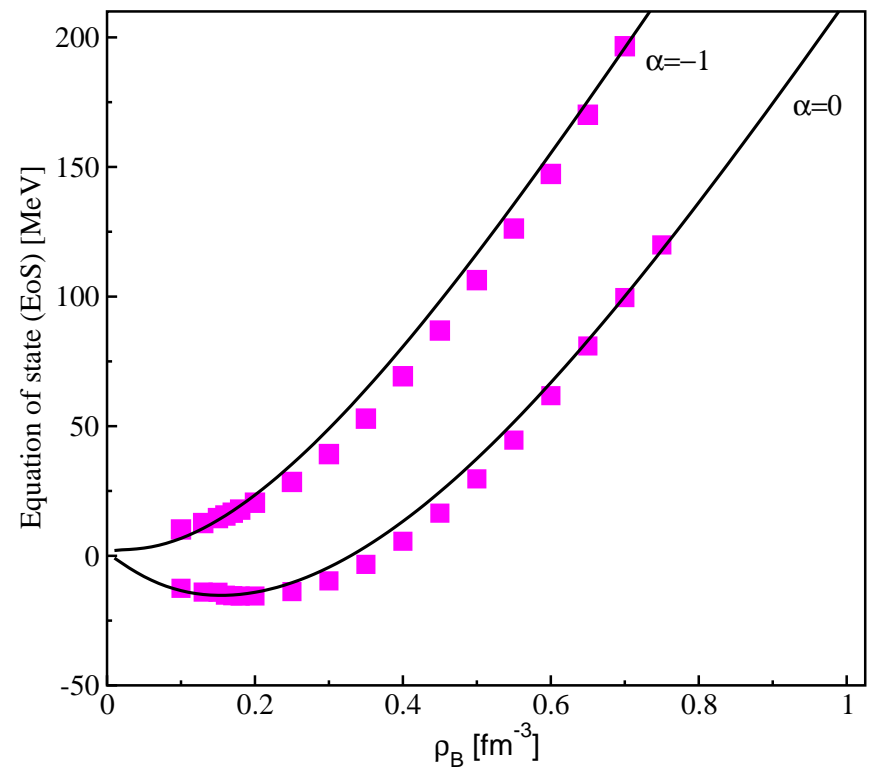

FIG. 2. Equation of state (EoS) in terms of the binding energy per nucleon as function of the baryon density $\rho_{B}$ for isospin-symmetric ( $\alpha=0$, lower curve and symbols) and pure neutron matter $(\alpha=-1$, upper curve and upper symbols). The NLD results (solid curves) are compared with DBHF calculations (filled squares) [38].

energy per nucleon as function of baryon density for isospinsymmetric $(\alpha=0)$ and pure neutron matter $(\alpha=-1)$. The isospin-asymmetry parameter $\alpha$ is defined in the usual way as $\alpha=\frac{J_{p}^{0}-J_{n}^{0}}{J_{p}^{0}+J_{n}^{0}}$, where $J_{p, n}^{0}$ denote the proton and neutron densities, respectively. The momentum-dependent monopole form-factor of the NLD model regulates the high-density dependence of the fields such that the NLD EoS fits very well the DBHF calculations, for both, symmetric nuclear and pure neutron matters. Note that the NLD parameters are not fitted to the calculations of DBHF models, but to the empirical information at ground state density only.

In Fig. 3 the density dependence of the EoS is displayed again, but now we compare the NLD calculation with other RMF models, which have been widely used in studies of finite nuclei and of nuclear matter. Even if all models give similar results for the saturation point of nuclear matter, the differences between the NLD model and the other RMF approaches at densities beyond the ground state are large. In particular, the NL3* parametrization of Lalazissis et. al. [37], which is based on the Walecka model with non-linear selfinteractions predicts the stiffest EoS, while the RMF approaches DD and $\mathrm{D}^{3} \mathrm{C}$ with density-dependent meson-nucleon couplings give a softer density behavior at high densities. However, none of these RMF approaches can reproduce the microscopic calculations of the DBHF model at such high densities. In general, the non-linear density dependence of the NLD model, induced by the cut-off form factor, result in a much softer EoS at high densities, and it is in agreement with the DBHF calculations. Note that heavy-ion studies at energies just below the kaon production threshold $\left(E_{\text {beam }} \leq 1 \mathrm{GeV} / A\right)$ on collective nucleon flows [48-50] and on produced mesons (positive 


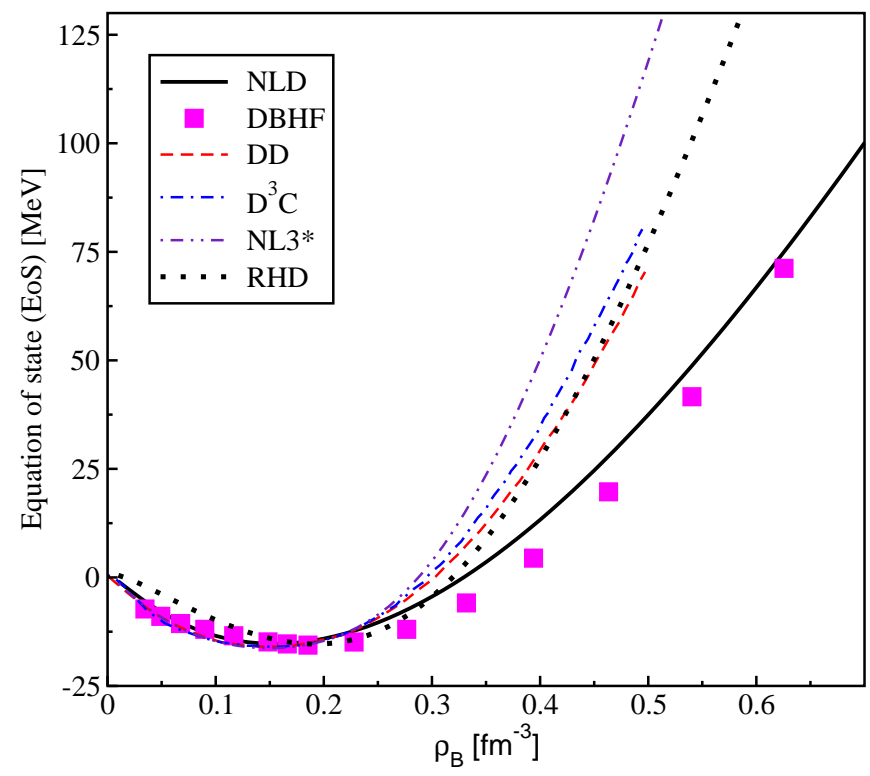

FIG. 3. Same as in Fig. 2, but now the NLD model (solid curve) is compared with various RMF calculations. These are the NL3* parametrization (dashed-dotted-dotted curve) [37], the density-dependent (DD, dashed curve) and derivative-coupling $\left(\mathrm{D}^{3} \mathrm{C}\right.$, dashed-dotted curve) models $[19,22]$ and the linear Walecka model (RHD, dotted curve) [3]. The DBHF calculations (filled squares) are shown too. All the calculations refer to isospinsymmetric $(\alpha=0)$ nuclear matter.

charged kaons) [51-54] support a rather soft EoS at densities around $\rho_{B} \simeq(2-3) \rho_{\text {sat }}$.

Furthermore, an interesting issue concerns the inclusion of the non-linear selfinteractions of the $\sigma$-field in the RMF descriptions. It is well known, that such terms may cause divergences at very high densities, where one intends to study compact neutron stars. Indeed, many RMF approaches such as the NL3* [37] or the NL $\rho$ and NL $\rho \delta$ [55] parameterizations, lead to a non-physical behavior of the $\sigma$-field at supra-normal densities. This is not the case in the NLD model, where the non-linear selfinteraction terms of the $\sigma$-meson does not cause any divergences of the $\sigma$-field even at supra-normal densities. This novel NLD feature arises from the suppression of the scalar sources by the cut-off function $\mathcal{D}$, which in the limiting case of large densities $\rho_{B}$ tend to vanishes.

Another quantity of interest is the symmetry energy. This quantity is important for astrophysical applications, since it directly influences the density dependence of the protonfraction in the neutron star interior. It is extracted as the difference between the EoS's from pure neutron and symmetric matters. Fig. 4 shows the symmetry energy as function of the baryon density in the NLD model, in other RMF models and in the DBHF approach. Note that the NLD results differ from the previous works [23]. This is because now the NLD model consistently includes the renormalization of the spinors. This is essential for the thermodynamic consistency of the formalism, which was not taken into account in Ref. [23]. Furthermore, a different form of the regulator is used here and the parameters of the NLD model are fitted us-

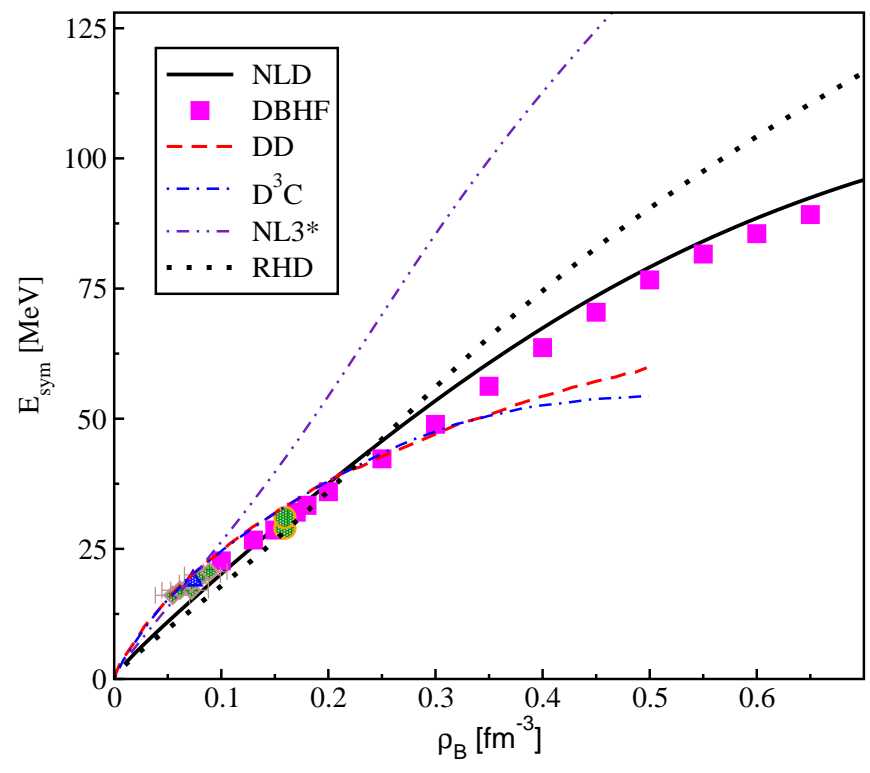

FIG. 4. Same as in Fig. 3, but now for the symmetry energy $E_{\text {sym }}$ as function of baryon density $\rho_{B}$. The additional symbols (diamonds, triangle and circles) around and below the saturation density refer to empirical data [56, 57].

ing low-density observables at saturation. At first, all models describes fairly well the empirically known region around the saturation density, as it was already shown in Table I. However, the differences between the models become more pronounced with increasing density. The most stiffer symmetry energy is obtained again in the NL3* and the RHD calculations, because in these cases $E_{\text {sym }}$ is just proportional to the $\rho$-meson, which linearly increases with density. Furthermore, the symmetry energy is considerably softened in the DD and $\mathrm{D}^{3} \mathrm{C}$ approaches, due to the exponentially decreasing density dependence of the $\rho$-nucleon vertices. On the other hand, the NLD model leads to a softer density behavior of the symmetry energy relative to the standard NL and RHD parametrization but to a stiffer dependence than the density-dependent approaches. It is again an interesting feature that the NLD calculations predict almost perfectly the calculations of the microscopic DBHF calculations [38].

\section{In-medium nucleon optical potentials}

Apart the density dependence, which arises from both, the source terms of the meson-field equations and the cut-off functions, the NLD selfenergies depend explicitly on momentum. The momentum (or energy) behavior of the RMF fields is described by the in-medium Schrödinger-equivalent optical potential. This quantity results from the reduction of the Dirac equation and reads [58]

$$
U_{\text {opt }}=-S+\frac{E}{m} V+\frac{1}{2 m}\left(S^{2}-V^{2}\right),
$$

where the selfenergies $S=\Sigma_{s i}\left(\rho_{B}, p\right), V=\Sigma_{v i}^{0}\left(\rho_{B}, p\right)$ refer to a proton $(i=p)$ or a neutron $(i=n)$ with a particular 


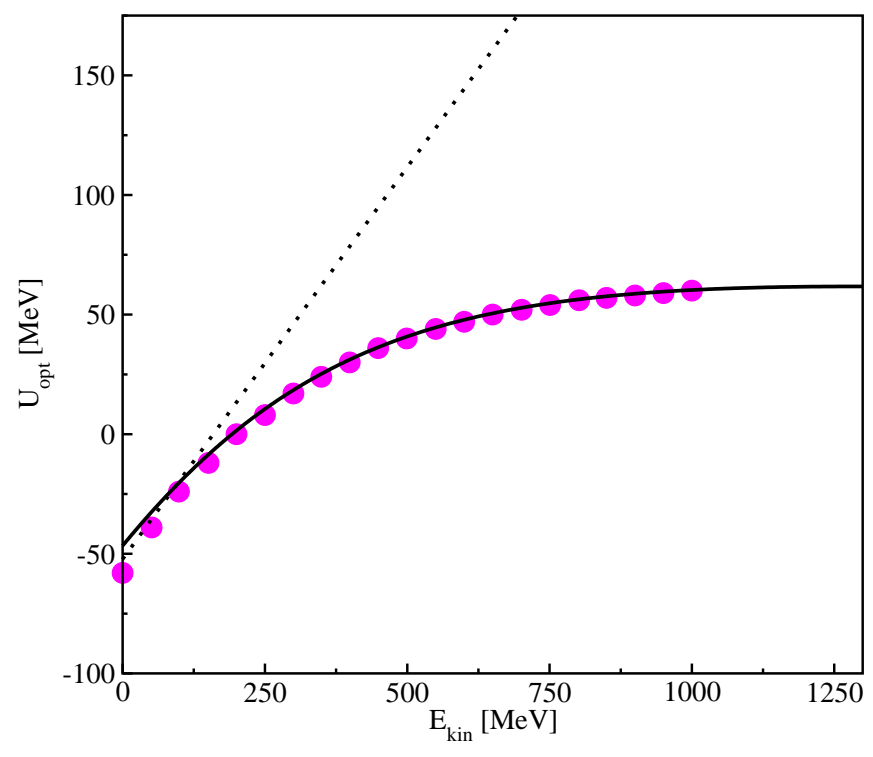

FIG. 5. (Color online) The in-medium proton optical potential $U_{\text {opt }}$ according Eq. (71) as function of the in-medium single-particle kinetic energy, Eq. (72), within the NLD model (solid curve) and the the RHD [3] approach at saturation density. The symbols (filled circles) refers to the results of the Dirac phenomenology [5, 6].

momentum $|\vec{p}|=p$ relative to nuclear matter at rest at a given density $\rho_{B}$ and isospin-asymmetry $\alpha$. The single-particle energy $E$ is calculated from the in-medium dispersion relation

$$
E=\sqrt{m^{* 2}+p^{2}}+V
$$

and the kinetic energy reads $E_{k i n}=E-m[9,22,31]$. Here we consider the real part of $U_{o p t}$ only, which enters the momentum dependent mean-field dynamics of a nucleon in nuclear matter. The imaginary part, which is beyond the scope of the present work, can be further accounted for in the collision integral within the relativistic transport equation, see for instance Ref. [12], when applying the NLD approach to the description of heavy-ion collisions. Alternatively, one can use a dispersion theoretical framework to calculate the imaginary part of the optical potential using as an input the real part of the optical potential in the dispersion integral, see Ref. [24] as an example of such approach to the imaginary part of $U_{\text {opt }}$. In this context we would like to note, that the imaginary contributions of the relativistic mean-fields do not influence essentially the real part of the optical potential, see for example Refs. [7, 22]. The reason is, that the imaginary contributions of the selfenergies, as obtained from empirical studies, are of similar magnitude. They enter only through the terms quadratic in the selfenergies in the expression for the real part of the Schrödinger-equivalent optical potential, Eq. (71), and therefore they almost cancel each other.

Fig. 5 shows the results of our calculations in comparison with the empirical data extracted from Dirac phenomenology $[5,6]$ for the in-medium proton optical potential in symmetric $(\alpha=0)$ nuclear matter at saturation density $\rho_{B}=\rho_{\text {sat }}$. The optical potential saturates with increasing single-particle energy in the NLD model and it is in agreement with the

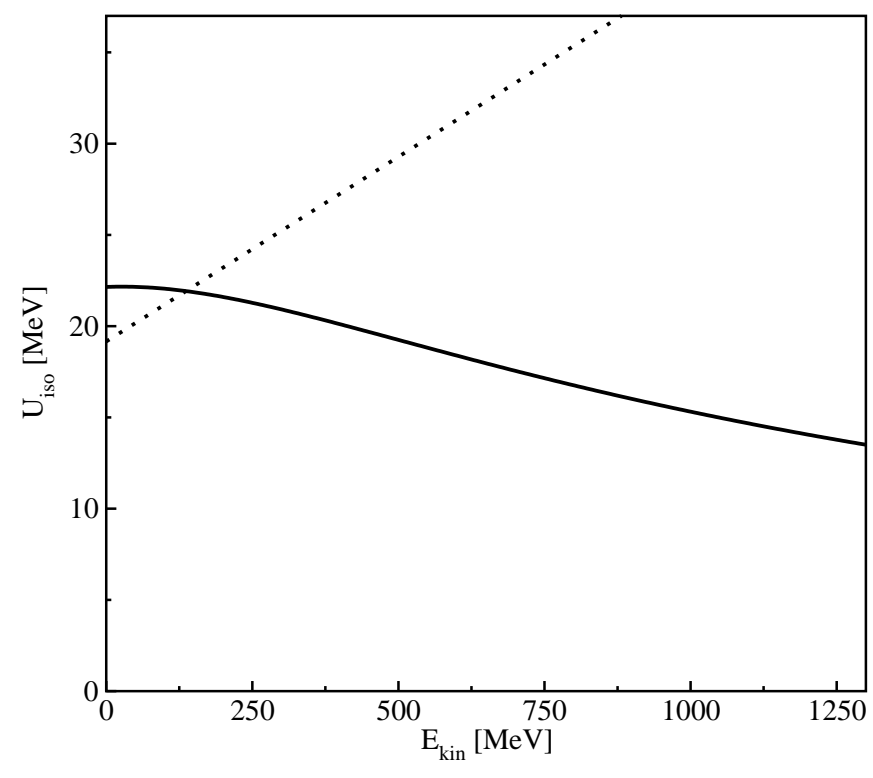

FIG. 6. Energy dependence of the Lane-type optical potential $U_{i s o}$, Eq. (73), for asymmetric $(\alpha=0.4)$ nuclear matter at saturation density. Calculation in the RHD [3] (dotted curve) and NLD (solid curve) models are shown.

experimental data. The saturation mechanism is induced by the explicit momentum dependence of the cut-off functions, which drop with increasing momentum.

It is a novel feature of the NLD model, that the regulators with a simple momentum-dependent monopole form are sufficient to describe accurately the bulk properties of nuclear matter and at the same time the empirical energy dependence of the optical potential. In fact, this issue has been a long-standing problem in nuclear matter studies, when one attempted to describe heavy-ion reactions within RMF models [59]. As also shown in Fig. 5, in standard RMF models, such as the widely used NL-parameterizations [37, 60], which describe excellent the saturation properties and also the properties of finite nuclei, cannot reproduce the correct energy dependence of the optical potential. Moreover, they strongly diverge with increasing energy. Similar conclusions are obtained in the RMF approaches with density-dependent vertex functions [19], except if one includes additional energydependent terms [22]. Note that, the microscopic DBHF models [9, 31, 32] describe satisfactory the empirical data at low energies bellow the pion production threshold.

For isospin-asymmetric nuclear matter the key quantity is the so-called Lane potential [46, 61], which is defined as the difference between the neutron and proton optical potentials

$$
U_{i s o}=\frac{U_{n}-U_{p}}{2|\alpha|} .
$$

Fig. 6 displays the energy dependence of the Lane potential. In contrast to the case of isospin-symmetric matter, empirical information is here less known. The studies from Ref. [46] predict a decrease of the Lane potential with increasing momentum, but other analysis [61] conclude the opposite trend 


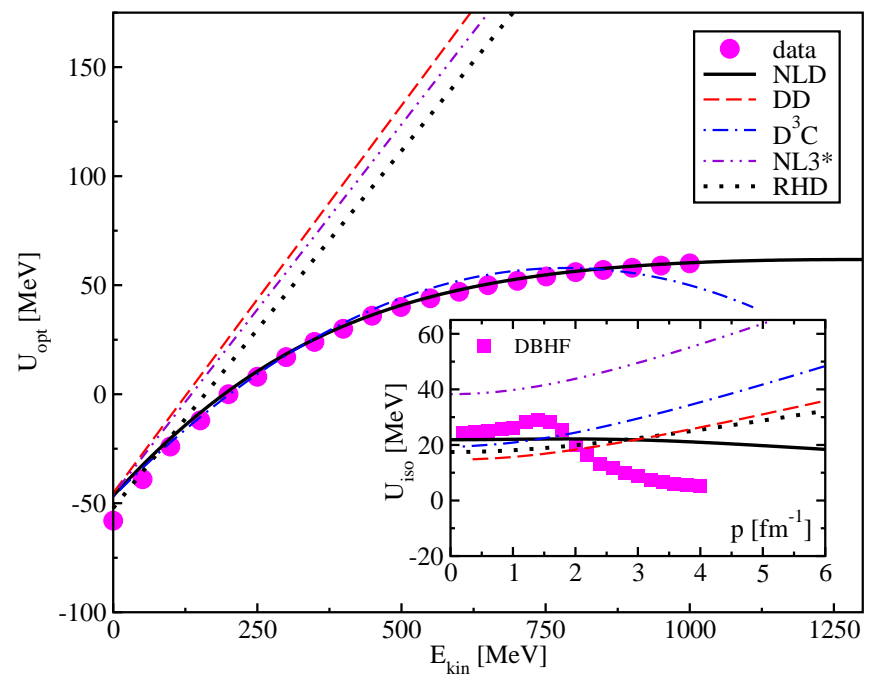

FIG. 7. Energy (momentum) dependence of the optical $U_{\text {opt }}$ (Lane) potentials in the main (inserted) panel. The curves have the same meaning as in Figs. 3 and 4 . Here the $\mathrm{D}^{3} \mathrm{C}$ results are taken from [64].

with the result of an increasing Lane potential. The microscopic DBHF calculations predict also a decreasing optical potential, which is in agreement with results of other BHF calculations $[62,63]$. Furthermore, the standard RHD model leads to an almost quadratic (or linear in energy) dependence in momentum, just because of the missing momentum dependence in the RHD selfenergies (similar results are obtained in the $\mathrm{NL}^{*}$ and DD parameterizations). The NLD calculations predict a weakly decreasing Lane potential, which is in qualitative agreement with the (D)BHF results.

We compare now the NLD results separately in Fig. 7 for both, the in-medium proton optical potential for symmetric nuclear matter and the Lane potential, with the same RMF approaches as in Figs. 3 and 4. Not only the original linear Walecka model (RHD), but also more modern RMF approaches, such as the non-linear NL3 model in its updated version (NL3*) and the density-dependent RMF model (DD) predict an energy dependence, which is not consistent with phenomenology (filled circles). This circumstance was improved in a modified DD model $\left(\mathrm{D}^{3} \mathrm{C}\right)$ by the inclusion of terms linearly proportional to the single-particle energy. The NLD model provides here a smoother energy dependence.

The situation for the Lane potential is shown in the insert of Fig. 7. The interesting issue here is, that not only the standard RMF models (RHD, NL3* and DD), but also the energydependent RMF approach $\left(\mathrm{D}^{3} \mathrm{C}\right)$, show a common behavior in momentum. This is due to the fact that the isovector channel in the $\mathrm{D}^{3} \mathrm{C}$ RMF model does not include any explicit momentum dependence [64]. On the other hand, in the NLD model also the isovector mean-field depends on momentum and predicts a decrease of the Lane potential with increasing momentum. This NLD trend seems to be supported by microscopic DBHF (filled symbols in the insert of Fig. 7), at least on a qualitative level.

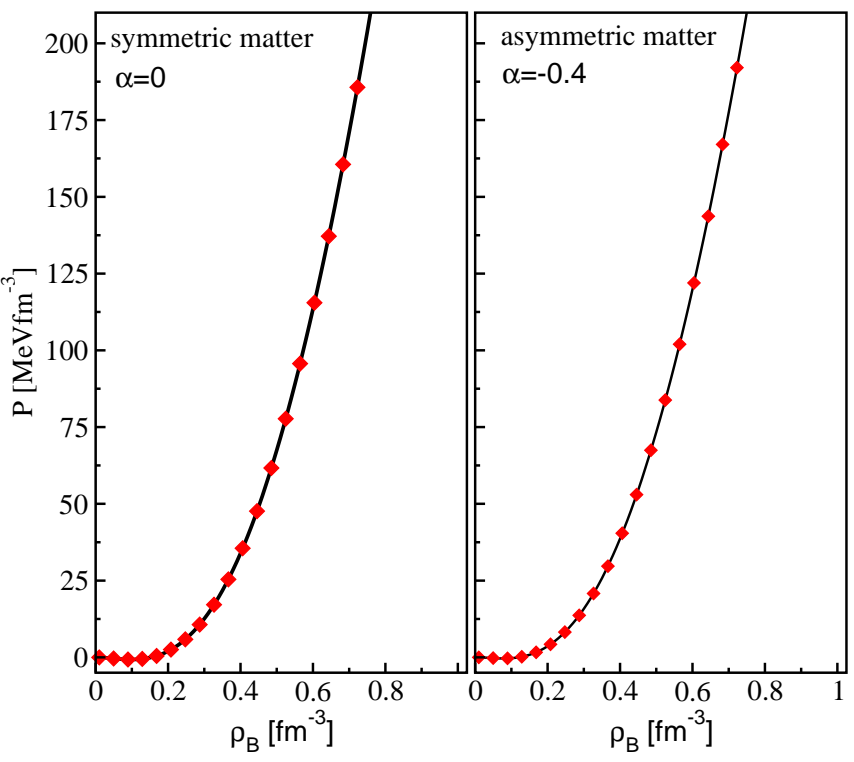

FIG. 8. Thermodynamic consistency of the NLD model for pure symmetric nuclear $(\alpha=0$, left panel) and asymmetric $(\alpha=-0.4$, right panel) matters. The pressure densities $P$ as function of the baryon density $\rho_{B}$ are shown, as calculated within the perfect fluid formula (solid curves) using the 1.h.s. of Eq. (76) and from the thermodynamic definition (filled diamonds) using the r.h.s. of Eq. (76).

\section{Thermodynamic consistency of NLD model}

As showed by Weisskopf [30] in an independent-particle model, the single particle energy at the Fermi surface must be equal the average energy per particle at saturation density. Hugenholtz and Van Hove [14] proved this also for an interacting Fermi gas at zero temperature.

For the thermodynamic consistency of the RMF model it is sufficient to prove Euler's theorem

$$
\varepsilon=-P+\sum_{i=p, n} \mu_{i} \rho_{B_{i}},
$$

with the chemical potentials $\mu_{i}=\frac{\partial \varepsilon}{\partial \rho_{B_{i}}}$ and the thermodynamic definition of the pressure

$$
P=\rho_{B}^{2} \frac{\partial\left(\varepsilon / \rho_{B}\right)}{\partial \rho_{B}} .
$$

The expression in Eq. (74) allow to examine the internal consistency of the model. For this purpose it is sufficient to check the equality between the pressure obtained from the assumption of nuclear matter as a prefect-fluid system and the pressure obtained from the thermodynamic definition. That is

$$
P=\frac{1}{3}\left(T^{x x}+T^{y y}+T^{z z}\right)=\rho_{B}^{2} \frac{\partial\left(\varepsilon / \rho_{B}\right)}{\partial \rho_{B}},
$$

where $\varepsilon$ and $T^{i i}(i=x, y, z)$ denote the energy density and the spatial diagonal components of the energy-momentum tensor tensor $T^{\mu \nu}$, respectively. 


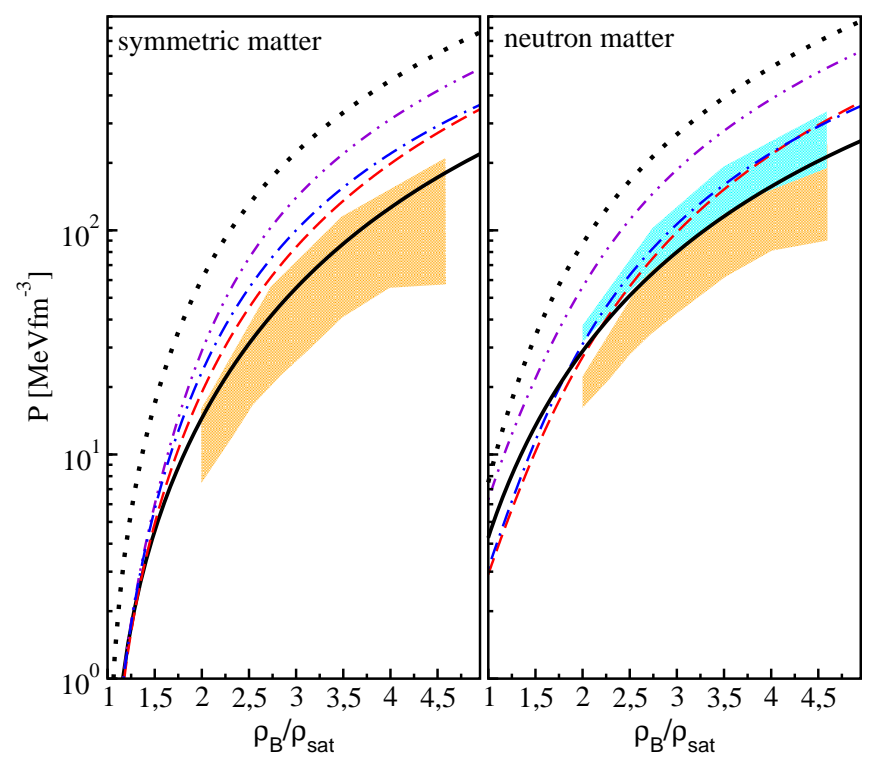

FIG. 9. Equation of state in terms of the pressure densities as function of the baryon density (normalized to the corresponding saturation values $\rho_{\text {sat }}$ ). The shaded areas denote possible experimental regions, as extracted from studies on heavy-ion collisions [48]. The different curves have the same meaning as in Figs. 3 and 4.

Conventional RMF approaches with bare meson-nucleon couplings or density-dependent meson-nucleon vertices are thermodynamically consistent $[4,21,60,65]$. However, in the case of explicit energy or momentum-dependent mean-fields the situation is more complex. In fact, as we have examined here, one has to take care of the proper renormalization of the Dirac fields. This issue was not taken into account in previous studies [25] resulting in a deviation between the 1.h.s. and r.h.s. of Eq. (76) by $\simeq 5-10 \%$ at high baryon densities.

We have checked that the NLD model presented here satisfies thermodynamic consistency exactly for any kind of energy- or momentum-dependent cut-off form factors. This is demonstrated in Fig. 8 for the monopole form factor, where the pressure as function of density for symmetric nuclear matter (left panel) and pure neutron matter (right panel) are shown. Indeed, the pressures obtained from both definitions of Eq. (76) agree exactly. Furthermore, the chemical potentials at zero temperature are equal to the corresponding Fermi energies of protons and neutrons (not shown here) and therefore Euler's theorem is evidently fulfilled.

We can now consider the high-density behavior of the pressure by comparing our results with available empirical informations. Fig. 9 shows the density dependence of the pressure densities in symmetric matter and pure neutron matter. The conventional RHD approach leads again to a stiff behavior for all the densities. Similar conclusions are drawn for the NL3*, $\mathrm{DD}$ and $\mathrm{D}^{3} \mathrm{C}$ approaches for symmetric matter, where for pure neutron matter the density-dependent models come closer to the empirical HIC data. A more detailed discussion on these approaches can be found in Ref. [66]. The pressure in the NLD model show generally a softer density dependence and agree better with the estimated experimental regions, in par-

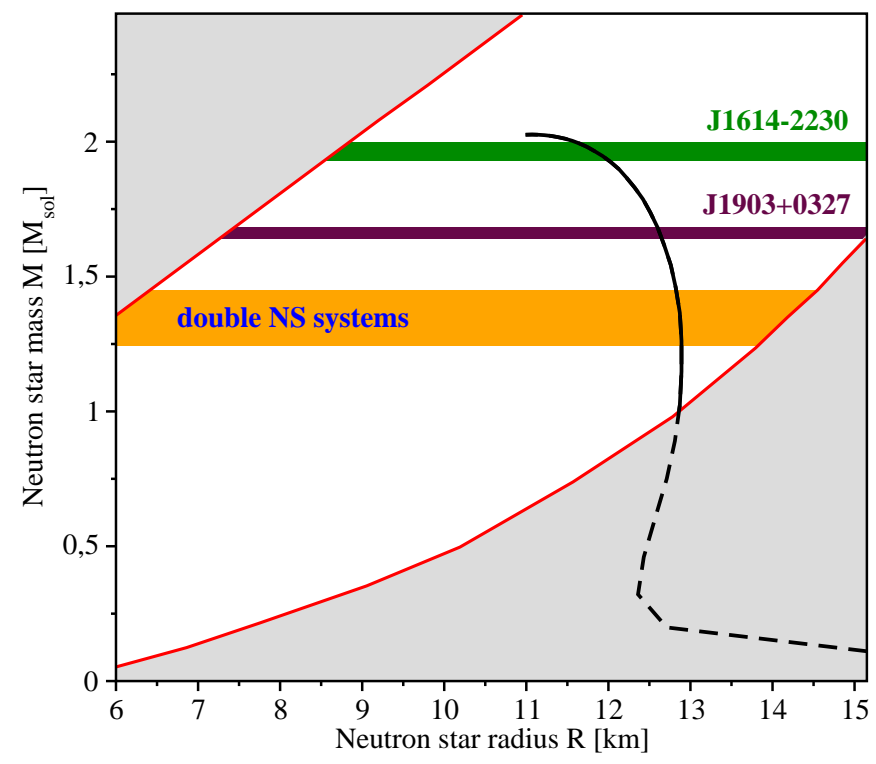

FIG. 10. Relation between Neutron star mass $M$ (in units of the solar mass $M_{\odot}$ ) versus radius $R$ in the NLD model (dashed and solid curves). The three horizontal shaded bands refer to astrophysical measurements from double neutron star (NS) systems [73, 74] and from the pulsars PSR J1903+0327 [75] and PSR J1614-2230 [67], as indicated. The other shaded areas bordered by thick curves indicate parameter space excluded by general relativity, causality (shaded area on the top-left) and rotational constraints (shaded area on the bottom-right) [73, 74].

ticular, at densities up to $\rho_{B} \simeq 4 \rho_{\text {sat }}$ for the symmetric case and for all densities for pure neutron matter. Note that for larger densities conclusions on the nuclear matter EoS from heavy-ion studies are more ambiguous, because at such high densities (or corresponding beam energies larger than $4 \mathrm{GeV}$ per nucleon) a large fraction of the initial energy is converted into new degrees of freedom $[48,66]$.

\section{E. High density observables and Neutron Stars}

We test now the high density domain of the NLD equation of state. Compact neutron stars offer such an opportunity to gain deeper insight into compressed baryonic matter. Of particular interest are recent measurements on the binary millisecond pulsar J1614-2230 with a mass of (1.97 \pm 0.04) $M_{\odot}$ [67]. The latter is much heavier than the average mass of the binary radio pulsars $M=1.35 \pm 0.04 M_{\odot}$ [68] and provides a strong constraint on high density EoS. Therefore, we apply the NLD model to spherical, non-rotating stars in $\beta$-equilibrium between protons, neutrons and electrons including crustal effects on the EoS [69]. The star structure is calculated by solving the Tolman-Oppenheimer-Volkov (TOV) equation [70-72].

The results for neutron stars are shown in Fig. 10 in terms of the mass-radius relation. The various astrophysical measurements of NS masses [73, 74] can be arranged in the three horizontal shaded areas as displayed in Fig. 10. The low- 


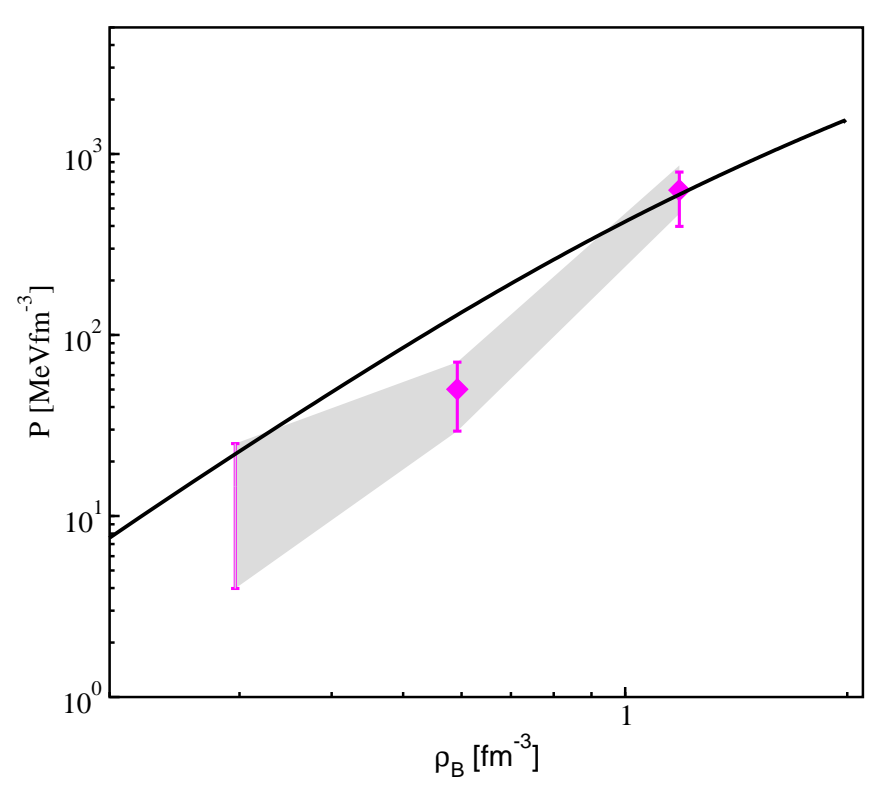

FIG. 11. Pressure densities as function of the baryon density for nuclear matter in $\beta$-equilibrium within the NLD (solid curve) model. The shaded areas together with the three error bars indicate the pressure-density region extracted directly from neutron-star measurements [26].

est band around an average value of $1.44 M_{\odot}$ refer to the well established measurements on double neutron star systems and the middle one around $1.67 M_{\odot}$ on the extracted mass of the pulsar PSR J1903+0327. The band on the top represents by far the highest precisely observed neutron star mass $1.97 \pm 0.04 M_{\odot}$ of the pulsar PSR J1614-2230 [67]. There are two regions in Fig. 10 excluded [73, 74] by general relativity, causality (shaded area on the top-left) and rotational constraints (shaded area on the bottom-right).

The neutron stars mass-radius relation in the NLD model is shown by the solid/dotted curve in Fig. 10. The dotted part of the NLD curve is excluded by rotational constraints. The solid curve crosses the low-mass constraints, and arrives to a maximum neutron star mass of $M=2.03 M_{\odot}$ at a radius of $R=11.07 \mathrm{~km}$ and a corresponding central density of $\rho_{c} \simeq$ $7 \rho_{\text {sat }}$. The NLD prediction for the maximum value of neutron star masses crosses also the constraint provided by the pulsar PSR J1614-2230, and therefore this recent mass measurement is accommodated by the NLD model.

Other possible constraints on the high-density EoS are obtained by statistical Bayesian analyses, which rely on neutron star measurements [26, 27]. They provide the most probable distribution of the equation of state, as shown in Fig. 11 (shaded area) for highly compressed matter in $\beta$-equilibrium. The RHD model (not shown in this figure) leads to a too stiff density dependence and it overestimates this empirical region particularly at high densities. The NLD calculations, where only nucleonic degrees of freedom are accounted for, describe fairly well the most probable region of the pressure at high densities. Note again that the parameters of the NLD model have been adjusted just to the saturation properties of nuclear matter and to the energy dependence of the in-medium pro- ton optical potential at saturation, without the consideration of any other high density observables in the fit procedure. We conclude here that the NLD describes well the available lowand also high-density constraints on EoS of nuclear matter and neutron stars.

\section{SUMMARY}

In summary, in the present work the generalized form of the energy-momentum tensor in the NLD model was derived which allowed us to consider different forms of the regulator functions in the NLD Lagrangian. The thermodynamic consistency of the NLD model was further demonstrated for arbitrary choice of the regulator functions. A thorough study of the properties of nuclear matter around saturation density has been performed. We have shown that the NLD approach describes well the saturation properties of the nuclear matter and compares remarkably well with microscopic calculations and Dirac phenomenology. We have investigated the high density part of the NLD EoS. This is relevant for the neutron stars in $\beta$-equilibrium. We found that the low density constraints imposed on the nuclear matter EoS and by the momentum dependence of the Schrödinger-equivalent optical potential lead to a maximum mass of the neutron stars around $M \simeq 2 M_{\odot}$. The latter mass accommodates the observed mass in the J16142230 millisecond radio pulsar system. We further studied the EoS of matter in $\beta$-equilibrium and find that the high density pressure-density diagram as extracted from astrophysical measurements can be well described in the NLD model which rely on nucleonic degrees of freedom only.

The EoS proposed here can be used in transport theoretical studies of nuclear collisions, since, it describes very well both, the low energy (density) and the high energy (density) regions of the nuclear phase diagram. The model predicts saturation of the optical potential at high energies and results in saturations of the symmetry energy. An interesting finding is that the momentum dependent interaction make the EoS softer at low densities, however, it is still stiff enough at supra-normal densities to account for the recent measurements of the neutron star masses. Furthermore, the model can be applied to the transport description of the anti-nucleon optical potential as well as to the study of dynamics of compressed matter in reactions induced by heavy-ions and anti-proton beams at the future FAIR facility.

\section{ACKNOWLEDGMENTS}

This work was supported by DFG and by DFG through TR16. We are greatful to Dr. Thomas von Chossy for discussions concerning the numerical implementation of the minimization routines. We also acknowledge the correspondence with Prof. Dr. Lie-Wen Chen and Bao-Jun Cai on the thermodynamic consistency of the NLD model. 


\section{Appendix A: Notations and infinetisemal variations}

We use following abbreviations for higher-order partial derivatives

$$
\begin{aligned}
\partial_{\alpha_{1} \cdots \alpha_{n}} & :=\partial_{\alpha_{1}} \partial_{\alpha_{2}} \cdots \partial_{\alpha_{n}}, \\
\partial^{\alpha_{1} \cdots \alpha_{n}} & :=\partial^{\alpha_{1}} \partial^{\alpha_{2}} \cdots \partial^{\alpha_{n}}, \\
\partial_{\alpha_{1} \cdots \alpha_{n}}^{\beta_{1} \cdots \beta_{m}} & :=\partial_{\alpha_{1}} \cdots \partial_{\alpha_{n}} \partial^{\beta_{1}} \cdots \partial^{\beta_{m}} .
\end{aligned}
$$

In the following appendices we will need various definitions of infinitesimal variations, which are specified here. The total variation of a field $\varphi_{r}(x)$ with $x^{\mu}=\left(x^{0}, \vec{x}\right)$ is defined as

$$
\delta_{T} \varphi_{r}(x):=\varphi_{r}^{\prime}\left(x^{\prime}\right)-\varphi_{r}(x)
$$

with the variation with respect to the 4-coordinate given by

$$
x^{\prime \mu}:=x^{\mu}+\delta x^{\mu}=x^{\mu}+\Delta \vartheta^{\mu \nu} x_{\nu}+\epsilon^{\mu},
$$

where $\delta x^{\mu}$ denotes an infinitesimal transformation, e.g., a constant translation, $\epsilon^{\mu}$, and/or a rotation, $\Delta \vartheta^{\mu \nu} x_{\mu}, \vartheta^{\mu \nu}$ is an infinitesimal antisymmetric tensor and $\Delta \vartheta^{\mu \nu}=-\Delta \vartheta^{\nu \mu}$. We define the infinitesimal transformation of the field at a fixed argument as

$$
\delta \varphi_{r}=\varphi_{r}^{\prime}-\varphi_{r}
$$

For the derivation of the Noether theorem we will need not only the infinitesimal variation of a field at fixed argument only, but also of its higher-order derivatives, i.e., $\delta\left(\partial_{\alpha_{1}} \varphi_{r}\right)$, $\delta\left(\partial_{\alpha_{1} \alpha_{2}} \varphi_{r}\right), \cdots, \delta\left(\partial_{\alpha_{1} \cdots \alpha_{n}} \varphi_{r}\right)$. For such variations the commutation between symbols $\delta$ and $\partial$ is holds, that is

$$
\begin{aligned}
\delta\left(\partial_{\alpha_{1}} \varphi_{r}\right) & =\partial_{\alpha_{1}} \varphi_{r}^{\prime}(x)-\partial_{\alpha_{1}} \varphi_{r}(x) \\
& =\partial_{\alpha_{1}}\left(\varphi_{r}^{\prime}(x)-\varphi_{r}(x)\right)=\partial_{\alpha_{1}} \delta \varphi_{r}(x), \\
\delta\left(\partial_{\alpha_{1} \alpha_{2}} \varphi_{r}\right) & =\partial_{\alpha_{1} \alpha_{2}} \varphi_{r}^{\prime}(x)-\partial_{\alpha_{1} \alpha_{2}} \varphi_{r}(x) \\
& =\partial_{\alpha_{1} \alpha_{2}}\left(\varphi_{r}^{\prime}(x)-\varphi(x)\right)=\partial_{\alpha_{1} \alpha_{2}}\left(\delta \varphi_{r}(x)\right)
\end{aligned}
$$

and obviously for higher-order fields. The total variation of a field $\varphi_{r}(x)$ can be written in a more handleable way as

$$
\begin{aligned}
\delta_{T} \varphi_{r}(x): & =\varphi_{r}^{\prime}\left(x^{\prime}\right)-\varphi_{r}(x) \\
& =\left[\varphi_{r}^{\prime}\left(x^{\prime}\right)-\varphi_{r}\left(x^{\prime}\right)\right]+\left[\varphi_{r}\left(x^{\prime}\right)-\varphi_{r}(x)\right],
\end{aligned}
$$

where the first term is just the variation $\delta \varphi_{r}$ at fixed argument. The second term in Eq. (A4) is the variation with respect to the argument. Eq. (A4) reduces in first order to

$$
\delta_{T} \varphi_{r}(x)=\delta \varphi_{r}(x)+\partial_{\alpha} \varphi_{r}(x) \delta x^{\alpha}
$$

\section{Appendix B: Derivation of the Noether theorem for higher-order Lagrangians}

For the derivation of the Noether theorem we start with the following Lagrangian density

$$
\mathcal{L}=\mathcal{L}\left[\varphi_{r}(x), \partial_{\alpha_{1}} \varphi_{r}(x), \cdots, \partial_{\alpha_{1} \cdots \alpha_{n}} \varphi_{r}(x)\right]
$$

Invariance of the Lagrangian density, (B1), with respect to an infinitesimal transformation of all the fields and their coordinates implies

$$
\mathcal{L}\left[\varphi_{r}^{\prime}\left(x^{\prime}\right), \partial_{\alpha_{1}}^{\prime} \varphi_{r}^{\prime}\left(x^{\prime}\right), \cdots, \partial_{\alpha_{1} \cdots \alpha_{n}}^{\prime} \varphi_{r}^{\prime}\left(x^{\prime}\right)\right]=\mathcal{L}\left[\varphi_{r}(x), \partial_{\alpha_{1}} \varphi_{r}(x), \cdots, \partial_{\alpha_{1} \cdots \alpha_{n}} \varphi_{r}(x)\right]
$$

In terms of the total variation Eq. (B2) results in

$$
\delta_{T} \mathcal{L}=\mathcal{L}\left[\varphi_{r}^{\prime}\left(x^{\prime}\right), \partial_{\alpha_{1}}^{\prime} \varphi_{r}^{\prime}\left(x^{\prime}\right), \cdots, \partial_{\alpha_{1} \cdots \alpha_{n}}^{\prime} \varphi_{r}^{\prime}\left(x^{\prime}\right)\right]-\mathcal{L}\left[\varphi_{r}(x), \partial_{\alpha_{1}} \varphi_{r}(x), \cdots, \partial_{\alpha_{1} \cdots \alpha_{n}} \varphi_{r}(x)\right]=0
$$

where $\partial_{\mu} \equiv \frac{\partial}{\partial x^{\mu}}$ and $\partial_{\mu}^{\prime} \equiv \frac{\partial}{\partial x^{\prime} \mu}$. Our goal is to arrive from Eq. (B3) to a continuity equation of the form

$$
\partial_{\mu} f^{\mu}=0
$$

with $f^{\mu}$ being a conserved current to be determined in the following. We will work out here all the analytical evaluations up to third order in the partial derivatives of the Lagrangian density, and we will give all the terms up to infinity in the final equations.

At first, the total variation of the Lagrangian density, Eq. (B3), can be written as

$$
\begin{aligned}
\delta_{T} \mathcal{L} & =\mathcal{L}\left[\varphi_{r}^{\prime}\left(x^{\prime}\right), \partial_{\alpha} \varphi_{r}^{\prime}\left(x^{\prime}\right), \partial_{\alpha \beta} \varphi_{r}^{\prime}\left(x^{\prime}\right), \ldots\right]-\mathcal{L}\left[\varphi_{r}\left(x^{\prime}\right), \partial_{\alpha} \varphi_{r}\left(x^{\prime}\right), \partial_{\alpha \beta} \varphi_{r}\left(x^{\prime}\right), \ldots\right] \\
& +\mathcal{L}\left[\varphi_{r}\left(x^{\prime}\right), \partial_{\alpha} \varphi_{r}\left(x^{\prime}\right), \partial_{\alpha \beta} \varphi_{r}\left(x^{\prime}\right), \ldots\right]-\mathcal{L}\left[\varphi_{r}(x), \partial_{\alpha} \varphi_{r}(x), \partial_{\alpha \beta} \varphi_{r}(x), \ldots\right]=0,
\end{aligned}
$$

where we used $\partial_{\mu}^{\prime} \varphi^{\prime}\left(x^{\prime}\right)=\partial_{\nu} \varphi^{\prime}\left(x^{\prime}\right) \partial_{\mu}^{\prime} x^{\nu}=\partial_{\nu} \varphi^{\prime}\left(x^{\prime}\right) \delta_{\mu}^{\nu}=\partial_{\mu} \varphi^{\prime}\left(x^{\prime}\right)$ where $\delta_{\mu}^{\nu}$ is a Kronecker symbol. The first line in Eq. (B5) is just the variation of $\mathcal{L}$ with respect to the fields $\varphi_{r}\left(r^{\prime}\right), \partial_{\alpha} \varphi_{r}\left(r^{\prime}\right), \cdots$ at fixed argument, whereas the terms in the second 
line give the variation of the Lagrangian with respect to the argument $x^{\mu}$. Since we consider infinitesimal transformations only, it is sufficient to evaluate latter quantity up to first order with respect to the argument. Therefore, Eq. (B5) can be written as

$$
\delta_{T} \mathcal{L}=\delta \mathcal{L}+\partial_{\alpha} \mathcal{L} \delta x^{\alpha}=0
$$

The variation at fixed argument, $\delta \mathcal{L}$, can be evaluated in the usual way according

$$
\delta \mathcal{L}=\sum_{r}\left[\frac{\partial \mathcal{L}}{\partial \varphi_{r}} \delta \varphi_{r}+\frac{\partial \mathcal{L}}{\partial\left(\partial_{\alpha_{1}} \varphi_{r}\right)} \delta\left(\partial_{\alpha_{1}} \varphi_{r}\right)+\frac{\partial \mathcal{L}}{\partial\left(\partial_{\alpha_{1} \alpha_{2}} \varphi_{r}\right)} \delta\left(\partial_{\alpha_{1} \alpha_{2}} \varphi_{r}\right)+\frac{\partial \mathcal{L}}{\partial\left(\partial_{\alpha_{1} \alpha_{2} \alpha_{3}} \varphi_{r}\right)} \delta\left(\partial_{\alpha_{1} \alpha_{2} \alpha_{3}} \varphi_{r}\right)+\cdots\right]
$$

Replacing the first term in Eq. (B7) with the help of the Euler-Lagrange equations of motion, Eq. (7), results in

$$
\begin{aligned}
\delta \mathcal{L}=\sum_{r} & {\left[\partial_{\alpha_{1}} \frac{\partial \mathcal{L}}{\partial\left(\partial_{\alpha_{1}} \varphi_{r}\right)} \delta \varphi_{r}-\partial_{\alpha_{1} \alpha_{2}} \frac{\partial \mathcal{L}}{\partial\left(\partial_{\alpha_{1} \alpha_{2}} \varphi_{r}\right)} \delta \varphi_{r}+\partial_{\alpha_{1} \alpha_{2} \alpha_{3}} \frac{\partial \mathcal{L}}{\partial\left(\partial_{\alpha_{1} \alpha_{2} \alpha_{3}} \varphi_{r}\right)} \delta \varphi_{r}-\cdots\right.} \\
& \left.+\frac{\partial \mathcal{L}}{\partial\left(\partial_{\alpha_{1}} \varphi_{r}\right)} \delta\left(\partial_{\alpha_{1}} \varphi_{r}\right)+\frac{\partial \mathcal{L}}{\partial\left(\partial_{\alpha_{1} \alpha_{2}} \varphi_{r}\right)} \delta\left(\partial_{\alpha_{1} \alpha_{2}} \varphi_{r}\right)+\frac{\partial \mathcal{L}}{\partial\left(\partial_{\left.\alpha_{1} \alpha_{2} \alpha_{3} \varphi_{r}\right)}\right.} \delta\left(\partial_{\alpha_{1} \alpha_{2} \alpha_{3}} \varphi_{r}\right)+\cdots\right]
\end{aligned}
$$

We use the commutation between the variation at fixed argument and the partial derivative (see appendix A) and apply once the product rule as follows for the term proportional to $\partial_{\alpha_{1} \alpha_{2}} \delta \varphi$

$$
\frac{\partial \mathcal{L}}{\partial\left(\partial_{\alpha_{1} \alpha_{2}} \varphi_{r}\right)} \partial_{\alpha_{1} \alpha_{2}} \delta \varphi_{r}=-\partial_{\alpha_{1}} \frac{\partial \mathcal{L}}{\partial\left(\partial_{\alpha_{1} \alpha_{2}} \varphi_{r}\right)} \partial_{\alpha_{2}} \delta \varphi_{r}+\partial_{\alpha_{1}}\left[\frac{\partial \mathcal{L}}{\partial\left(\partial_{\alpha_{1} \alpha_{2}} \varphi_{r}\right)} \partial_{\alpha_{2}} \delta \varphi_{r}\right]
$$

For the term proportional to $\partial_{\alpha_{1} \alpha_{2} \alpha_{3}} \delta \varphi$ we apply the product rule twice

$$
\begin{aligned}
\frac{\partial \mathcal{L}}{\partial\left(\partial_{\alpha_{1} \alpha_{2} \alpha_{3}} \varphi_{r}\right)} \partial_{\alpha_{1} \alpha_{2} \alpha_{3}} \delta \varphi_{r}= & \partial_{\alpha_{1} \alpha_{2}} \frac{\partial \mathcal{L}}{\partial\left(\partial_{\left.\alpha_{1} \alpha_{2} \alpha_{3} \varphi_{r}\right)} \partial_{\alpha_{3}} \delta \varphi_{r}\right.} \\
& -\partial_{\alpha_{2}}\left[\partial_{\alpha_{1}} \frac{\partial \mathcal{L}}{\partial\left(\partial_{\left.\alpha_{1} \alpha_{2} \alpha_{3} \varphi_{r}\right)}\right.} \partial_{\alpha_{3}} \delta \varphi_{r}\right]+\partial_{\alpha_{1}}\left[\frac{\partial \mathcal{L}}{\partial\left(\partial_{\left.\alpha_{1} \alpha_{2} \alpha_{3} \varphi_{r}\right)} \partial_{\alpha_{2} \alpha_{3}} \delta \varphi_{r}\right]}\right.
\end{aligned}
$$

and so forth for the terms proportional to higher-order derivatives. In total, this procedure leads to a series of terms proportional to a 4-divergences only. It is more convenient to arrange these terms such to obtain several infinite series for each derivative field $\delta \varphi, \partial_{\alpha_{1}} \delta \varphi, \partial_{\alpha_{1} \alpha_{2}} \delta \varphi, \partial_{\alpha_{1} \cdots \alpha_{n}} \delta \varphi$. After insertion of Eqs. (B9) and (B10) into the Eq. (B8) and after their rearrangement we obtain as an intermediate result

$$
\begin{aligned}
& \delta \mathcal{L}=\partial_{\mu}\left\{\left[\frac{\partial \mathcal{L}}{\partial\left(\partial_{\mu} \varphi_{r}\right)}-\partial_{\alpha_{1}} \frac{\partial \mathcal{L}}{\partial\left(\partial_{\mu \alpha_{1}} \varphi_{r}\right)}+\partial_{\alpha_{1} \alpha_{2}} \frac{\partial \mathcal{L}}{\partial\left(\partial_{\left.\mu \alpha_{1} \alpha_{2} \varphi_{r}\right)}\right.}-\cdots+(-)^{n} \partial_{\alpha_{1} \cdots \alpha_{n}} \frac{\partial \mathcal{L}}{\partial\left(\partial_{\mu \alpha_{1} \cdots \alpha_{n}} \varphi_{r}\right)}\right] \delta \varphi_{r}\right. \\
& +\left[\frac{\partial \mathcal{L}}{\partial\left(\partial_{\mu \sigma_{1}} \varphi_{r}\right)}-\partial_{\alpha_{1}} \frac{\partial \mathcal{L}}{\partial\left(\partial_{\left.\mu \sigma_{1} \alpha_{1} \varphi_{r}\right)}\right.}+\cdots+(-)^{n} \partial_{\alpha_{1} \cdots \alpha_{n}} \frac{\partial \mathcal{L}}{\partial\left(\partial_{\mu \sigma_{1} \alpha_{1} \cdots \alpha_{n}} \varphi_{r}\right)}\right] \partial_{\sigma_{1}} \delta \varphi_{r} \\
& +\left[\frac{\partial \mathcal{L}}{\partial\left(\partial_{\left.\mu \sigma_{1} \sigma_{2} \varphi_{r}\right)}\right.}+\cdots+(-)^{n} \partial_{\alpha_{1} \cdots \alpha_{n}} \frac{\partial \mathcal{L}}{\partial\left(\partial_{\mu \sigma_{1} \sigma_{2} \alpha_{1} \cdots \alpha_{n}} \varphi_{r}\right)}\right] \partial_{\sigma_{1} \sigma_{2}} \delta \varphi_{r} \\
& \left.+\left[\frac{\partial \mathcal{L}}{\partial\left(\partial_{\mu \sigma_{1} \cdots \sigma_{n}} \varphi_{r}\right)}-\partial_{\alpha_{1}} \frac{\partial \mathcal{L}}{\partial\left(\partial_{\left.\mu \sigma_{1} \cdots \sigma_{n} \alpha_{1} \varphi_{r}\right)}\right.}+\cdots+(-)^{n} \partial_{\alpha_{1} \cdots \alpha_{n}} \frac{\partial \mathcal{L}}{\partial\left(\partial_{\mu \sigma_{1} \cdots \sigma_{n} \alpha_{1} \cdots \alpha_{n}} \varphi_{r}\right)}\right] \partial_{\sigma_{1} \cdots \sigma_{n}} \delta \varphi_{r}\right\} .
\end{aligned}
$$

As next step we replace $\delta \varphi_{r}$ by the total variation, $\delta_{T} \varphi$, and insert the resulting equation into the total variation for the Lagrangian in Eq. (B6). Furthermore, we use

$$
\partial_{\alpha} \mathcal{L} \delta x^{\alpha}=\partial_{\mu}\left(g_{\alpha}^{\mu} \mathcal{L} \delta x^{\alpha}\right)
$$

where $g_{\alpha}^{\mu}$ is a metric tensor. Eq. (B12) obviously holds, when the infinitesimal transformation for the coordinates, $\delta x^{\mu}$, concerns a constant displacement of the 4 -vector $x^{\mu}$. In case of rotations, where $\delta x^{\mu}$ depends on the coordinate $x^{\mu}$ its self, Eq. (B12) still applies due to the antisymmetry of the tensor $\vartheta^{\mu \nu}$. These steps give us the final and general expression for the Noether theorem 
with respect to variations of the different fields and their coordinates

$$
\begin{aligned}
& \delta \mathcal{L}=\partial_{\mu}\left\{\left[\frac{\partial \mathcal{L}}{\partial\left(\partial_{\mu} \varphi_{r}\right)}-\partial_{\alpha_{1}} \frac{\partial \mathcal{L}}{\partial\left(\partial_{\mu \alpha_{1}} \varphi_{r}\right)}+\partial_{\alpha_{1} \alpha_{2}} \frac{\partial \mathcal{L}}{\partial\left(\partial_{\left.\mu \alpha_{1} \alpha_{2} \varphi_{r}\right)}\right.}-\cdots+(-)^{n} \partial_{\alpha_{1} \cdots \alpha_{n}} \frac{\partial \mathcal{L}}{\partial\left(\partial_{\mu \alpha_{1} \cdots \alpha_{n}} \varphi_{r}\right)}\right]\right. \\
& \times\left(\delta_{T} \varphi_{r}-\partial_{\alpha} \varphi_{r} \delta x^{\alpha}\right) \\
& +\left[\frac{\partial \mathcal{L}}{\partial\left(\partial_{\left.\mu \sigma_{1} \varphi_{r}\right)}\right.}-\partial_{\alpha_{1}} \frac{\partial \mathcal{L}}{\partial\left(\partial_{\left.\mu \sigma_{1} \alpha_{1} \varphi_{r}\right)}\right.}+\cdots+(-)^{n} \partial_{\alpha_{1} \cdots \alpha_{n}} \frac{\partial \mathcal{L}}{\partial\left(\partial_{\mu \sigma_{1} \alpha_{1} \cdots \alpha_{n}} \varphi_{r}\right)}\right] \\
& \times \partial_{\sigma_{1}}\left(\delta_{T} \varphi_{r}-\partial_{\alpha} \varphi_{r} \delta x^{\alpha}\right) \\
& +\left[\frac{\partial \mathcal{L}}{\partial\left(\partial_{\left.\mu \sigma_{1} \sigma_{2} \varphi_{r}\right)}\right.}+\cdots+(-)^{n} \partial_{\alpha_{1} \cdots \alpha_{n}} \frac{\partial \mathcal{L}}{\partial\left(\partial_{\left.\mu \sigma_{1} \sigma_{2} \alpha_{1} \cdots \alpha_{n} \varphi_{r}\right)}\right)}\right] \\
& \times \partial_{\sigma_{1} \sigma_{2}}\left(\delta_{T} \varphi_{r}-\partial_{\alpha} \varphi_{r} \delta x^{\alpha}\right) \\
& \vdots \\
& +\left[\frac{\partial \mathcal{L}}{\partial\left(\partial_{\mu \sigma_{1} \cdots \sigma_{n}} \varphi_{r}\right)}-\partial_{\alpha_{1}} \frac{\partial \mathcal{L}}{\partial\left(\partial_{\left.\mu \sigma_{1} \cdots \sigma_{n} \alpha_{1} \varphi_{r}\right)}\right.}+\cdots+(-)^{n} \partial_{\alpha_{1} \cdots \alpha_{n}} \frac{\partial \mathcal{L}}{\partial\left(\partial_{\mu \sigma_{1} \cdots \sigma_{n} \alpha_{1} \cdots \alpha_{n}} \varphi_{r}\right)}\right] \\
& \times \partial_{\sigma_{1} \cdots \sigma_{n}}\left(\delta_{T} \varphi_{r}-\partial_{\alpha} \varphi_{r} \delta x^{\alpha}\right) \\
& \left.-g^{\mu \alpha} \mathcal{L} \delta x_{\alpha}\right\}
\end{aligned}
$$

We consider now global phase transformations $(\epsilon \ll 1)$

$$
\delta x^{\mu}=0, \varphi_{r}^{\prime}\left(x^{\prime}\right)=e^{-i \epsilon} \varphi_{r}(x) \Rightarrow \delta \varphi_{r T}=\delta \varphi_{r}=-i \epsilon \varphi_{r}
$$

and obtain the following relations for global phase transformations

$$
\begin{aligned}
\delta \varphi_{r} & =-i \epsilon \varphi_{r}, \\
\partial_{\alpha_{1}} \delta \varphi_{r} & =-i \epsilon \partial_{\alpha_{1}} \varphi_{r}, \\
\partial_{\alpha_{1} \alpha_{2}} \delta \varphi_{r} & =-i \epsilon \partial_{\alpha_{1} \alpha_{2}} \varphi_{r}, \\
\cdots & , \\
\partial_{\alpha_{1} \alpha_{2} \cdots \alpha_{n}} \delta \varphi_{r} & =-i \epsilon \partial_{\alpha_{1} \alpha_{2} \cdots \alpha_{n}} \varphi_{r} .
\end{aligned}
$$

Therefore, the invariance of the Lagrangian under global phase transformations results to the continuity equation $\partial_{\mu} J^{\mu}=0$ with 
the current given by

$$
\begin{aligned}
J^{\mu}=- & i\left[\frac{\partial \mathcal{L}}{\partial\left(\partial_{\mu} \varphi_{r}\right)}-\partial_{\alpha_{1}} \frac{\partial \mathcal{L}}{\partial\left(\partial_{\mu \alpha_{1}} \varphi_{r}\right)}+\partial_{\alpha_{1} \alpha_{2}} \frac{\partial \mathcal{L}}{\partial\left(\partial_{\left.\mu \alpha_{1} \alpha_{2} \varphi_{r}\right)}\right.}-\cdots+(-)^{n} \partial_{\alpha_{1} \cdots \alpha_{n}} \frac{\partial \mathcal{L}}{\partial\left(\partial_{\mu \alpha_{1} \cdots \alpha_{n}} \varphi_{r}\right)}\right] \varphi_{r} \\
& +\left[\frac{\partial \mathcal{L}}{\partial\left(\partial_{\left.\mu \sigma_{1} \varphi_{r}\right)}\right.}-\partial_{\alpha_{1}} \frac{\partial \mathcal{L}}{\partial\left(\partial_{\left.\mu \sigma_{1} \alpha_{1} \varphi_{r}\right)}\right.}+\cdots+(-)^{n} \partial_{\alpha_{1} \cdots \alpha_{n}} \frac{\partial \mathcal{L}}{\partial\left(\partial_{\mu \sigma_{1} \alpha_{1} \cdots \alpha_{n}} \varphi_{r}\right)}\right] \partial_{\sigma_{1}} \varphi_{r} \\
& +\left[\frac{\partial \mathcal{L}}{\partial\left(\partial_{\left.\mu \sigma_{1} \sigma_{2} \varphi_{r}\right)}\right.}+\cdots+(-)^{n} \partial_{\alpha_{1} \cdots \alpha_{n}} \frac{\partial \mathcal{L}}{\partial\left(\partial_{\mu \sigma_{1} \sigma_{2} \alpha_{1} \cdots \alpha_{n}} \varphi_{r}\right)}\right] \partial_{\sigma_{1} \sigma_{2}} \varphi_{r} \\
& \vdots \\
& \left.+\left[\frac{\partial \mathcal{L}}{\partial\left(\partial_{\left.\mu \sigma_{1} \cdots \sigma_{n} \varphi_{r}\right)}\right.}-\partial_{\alpha_{1}} \frac{\partial \mathcal{L}}{\partial\left(\partial_{\left.\mu \sigma_{1} \cdots \sigma_{n} \alpha_{1} \varphi_{r}\right)}\right.}+\cdots+(-)^{n} \partial_{\alpha_{1} \cdots \alpha_{n}} \frac{\partial \mathcal{L}}{\partial\left(\partial_{\mu \sigma_{1} \cdots \sigma_{n} \alpha_{1} \cdots \alpha_{n}} \varphi_{r}\right)}\right] \partial_{\sigma_{1} \cdots \sigma_{n}} \varphi_{r}\right\} .
\end{aligned}
$$

Using Eqs. (10) for the tensors $\mathcal{K}_{r}^{\mu \sigma_{1} \sigma_{2} \cdots}$ one arrives to the Expression in Eq. (9).

The energy-momentum tensor is derived again with the help of Eq. (B13) for the case of constant 4-translations $x^{\prime \mu}:=$ $x^{\mu}+\delta x^{\mu}$. This means $\delta_{T} \varphi_{r}(x)=0$ and following expression is obtained

$$
\begin{aligned}
& \partial_{\mu}\left\{\left[\frac{\partial \mathcal{L}}{\partial\left(\partial_{\mu} \varphi_{r}\right)}-\partial_{\alpha_{1}} \frac{\partial \mathcal{L}}{\partial\left(\partial_{\left.\mu \alpha_{1} \varphi_{r}\right)}\right.}+\partial_{\alpha_{1} \alpha_{2}} \frac{\partial \mathcal{L}}{\partial\left(\partial_{\left.\mu \alpha_{1} \alpha_{2} \varphi_{r}\right)}\right.}-\cdots+(-)^{n} \partial_{\alpha_{1} \cdots \alpha_{n}} \frac{\partial \mathcal{L}}{\partial\left(\partial_{\mu \alpha_{1} \cdots \alpha_{n}} \varphi_{r}\right)}\right] \partial^{\nu} \varphi_{r}\right. \\
& \left.+\frac{\partial \mathcal{L}}{\partial\left(\partial_{\left.\mu \sigma_{1} \varphi_{r}\right)}\right.}-\partial_{\alpha_{1}} \frac{\partial \mathcal{L}}{\partial\left(\partial_{\left.\mu \sigma_{1} \alpha_{1} \varphi_{r}\right)}\right.}+\cdots+(-)^{n} \partial_{\alpha_{1} \cdots \alpha_{n}} \frac{\partial \mathcal{L}}{\partial\left(\partial_{\mu \sigma_{1} \alpha_{1} \cdots \alpha_{n}} \varphi_{r}\right)}\right] \partial_{\sigma_{1}}^{\nu} \varphi_{r} . \\
& \quad+\left[\frac{\partial \mathcal{L}}{\partial\left(\partial_{\left.\mu \sigma_{1} \sigma_{2} \varphi_{r}\right)}\right.}+\cdots+(-)^{n} \partial_{\alpha_{1} \cdots \alpha_{n}} \frac{\partial \mathcal{L}}{\partial\left(\partial_{\left.\mu \sigma_{1} \sigma_{2} \alpha_{1} \cdots \alpha_{n} \varphi_{r}\right)}\right)}\right] \partial_{\sigma_{1} \sigma_{2}}^{\nu} \varphi_{r} \\
& \quad \vdots \\
& +\left[\quad \frac{\partial \mathcal{L}}{\partial\left(\partial_{\left.\mu \sigma_{1} \cdots \sigma_{n} \varphi_{r}\right)}\right.}-\partial_{\alpha_{1}} \frac{\partial \mathcal{L}}{\partial\left(\partial_{\left.\mu \sigma_{1} \cdots \sigma_{n} \alpha_{1} \varphi_{r}\right)}\right.}+\cdots+(-)^{n} \partial_{\alpha_{1} \cdots \alpha_{n}} \frac{\partial \mathcal{L}}{\partial\left(\partial_{\mu \sigma_{1} \cdots \sigma_{n} \alpha_{1} \cdots \alpha_{n}} \varphi_{r}\right)}\right] \partial_{\sigma_{1} \cdots \sigma_{n}}^{\nu} \varphi_{r} \\
& \left.\quad-g^{\mu \nu} \mathcal{L}\right\} \delta x_{\nu}=0 .
\end{aligned}
$$

This leads to the continuity equation

$$
\partial_{\mu} T^{\mu \nu}=0
$$

with the energy-momentum tensor $T^{\mu \nu}$ given by

$$
\begin{aligned}
T^{\mu \nu}=- & \left\{\left[\frac{\partial \mathcal{L}}{\partial\left(\partial_{\mu} \varphi_{r}\right)}-\partial_{\alpha_{1}} \frac{\partial \mathcal{L}}{\partial\left(\partial_{\mu \alpha_{1}} \varphi_{r}\right)}+\partial_{\alpha_{1} \alpha_{2}} \frac{\partial \mathcal{L}}{\partial\left(\partial_{\left.\mu \alpha_{1} \alpha_{2} \varphi_{r}\right)}\right.}-\cdots+(-)^{n} \partial_{\alpha_{1} \cdots \alpha_{n}} \frac{\partial \mathcal{L}}{\partial\left(\partial_{\mu \alpha_{1} \cdots \alpha_{n}} \varphi_{r}\right)}\right] \partial^{\nu} \varphi_{r}\right. \\
& +\left[\frac{\partial \mathcal{L}}{\partial\left(\partial_{\left.\mu \sigma_{1} \varphi_{r}\right)}\right.}-\partial_{\alpha_{1}} \frac{\partial \mathcal{L}}{\partial\left(\partial_{\left.\mu \sigma_{1} \alpha_{1} \varphi_{r}\right)}\right.}+\cdots+(-)^{n} \partial_{\alpha_{1} \cdots \alpha_{n}} \frac{\partial \mathcal{L}}{\partial\left(\partial_{\mu \sigma_{1} \alpha_{1} \cdots \alpha_{n}} \varphi_{r}\right)}\right] \partial_{\sigma_{1}}^{\nu} \varphi_{r} \\
& +\left[\frac{\partial \mathcal{L}}{\partial\left(\partial_{\left.\mu \sigma_{1} \sigma_{2} \varphi_{r}\right)}\right.}+\cdots+(-)^{n} \partial_{\alpha_{1} \cdots \alpha_{n}} \frac{\partial \mathcal{L}}{\partial\left(\partial_{\mu \sigma_{1} \sigma_{2} \alpha_{1} \cdots \alpha_{n}} \varphi_{r}\right)}\right] \partial_{\sigma_{1} \sigma_{2}}^{\nu} \varphi_{r} \\
& \vdots \\
& +[ \\
& \left.-\left[\frac{\partial \mathcal{L}}{\partial\left(\partial_{\left.\mu \sigma_{1} \cdots \sigma_{n} \varphi_{r}\right)}^{\mu \nu} \mathcal{L} .\right.} \partial_{\alpha_{1}} \frac{\partial \mathcal{L}}{\partial\left(\partial_{\left.\mu \sigma_{1} \cdots \sigma_{n} \alpha_{1} \varphi_{r}\right)}\right.}+\cdots+(-)^{n} \partial_{\alpha_{1} \cdots \alpha_{n}} \frac{\partial \mathcal{L}}{\partial\left(\partial_{\left.\mu \sigma_{1} \cdots \sigma_{n} \alpha_{1} \cdots \alpha_{n} \varphi_{r}\right)}\right.}\right] \partial_{\sigma_{1} \cdots \sigma_{n}}^{\nu} \varphi_{r}\right\}
\end{aligned}
$$


Above expression for the energy-momentum tensor can be written also in a more compact form resulting to Eq. (12).

\section{Appendix C: Preliminaries for the NLD formalism}

For the derivation of the Dirac equation, the current and the energy-momentum tensor in the NLD model we need the derivatives of the NLD Lagrangian with respect to the spinor fields and their higher-order derivatives. We evaluate them here up to second order and provide all terms of infinite series in the final expressions. Since we are interesting on the derivatives with respect to the spinor fields only, we start with the NLD Lagrangian without the meson-field contributions. That is

$$
\begin{aligned}
\mathcal{L} & =\frac{1}{2}\left[\bar{\Psi} i \gamma_{\mu} \partial^{\mu} \Psi-\left(i \partial^{\mu} \bar{\Psi}\right) \gamma_{\mu} \Psi\right]-\bar{\Psi} \Psi m+\frac{g_{\sigma}}{2}[\bar{\Psi} \overleftarrow{\mathcal{D}} \Psi \sigma+\sigma \bar{\Psi} \overrightarrow{\mathcal{D}} \Psi]-\frac{g_{\omega}}{2}\left[\bar{\Psi} \overleftarrow{\mathcal{D}} \gamma^{\mu} \Psi \omega_{\mu}+\omega_{\mu} \bar{\Psi} \gamma^{\mu} \overrightarrow{\mathcal{D}} \Psi\right] \\
& -\frac{g_{\rho}}{2}\left[\bar{\Psi} \overleftarrow{\mathcal{D}} \gamma^{\mu} \vec{\tau} \Psi \vec{\rho}_{\mu}+\vec{\rho}_{\mu} \bar{\Psi} \vec{\tau} \gamma^{\mu} \overrightarrow{\mathcal{D}} \Psi\right]+\frac{g_{\delta}}{2}[\bar{\Psi} \overleftarrow{\mathcal{D}} \vec{\tau} \Psi \vec{\delta}+\vec{\delta} \bar{\Psi} \overrightarrow{\mathcal{D}} \Psi]
\end{aligned}
$$

The application of the various higher-order partial derivatives with respect to the spinor fields $\bar{\Psi}$ and $\Psi$ to the Lagrangian density in Eq. (C1) proceeds with the help of the multiple Taylor expansions, see Eqs. (26). It is convenient to rearrange these series in ascending order with respect to the partial derivatives. With $\vec{\xi}_{j}=-\zeta_{j}^{\alpha} i \vec{\partial}_{\alpha}$ and $\overleftarrow{\xi}_{j}=i \overleftarrow{\partial} \alpha \zeta_{j}^{\alpha}(j=1,2,3,4)$ where $\zeta_{j}^{\mu}=v_{j}^{\mu} / \Lambda$ one obtains for the expansion up to order $n$

$$
\begin{aligned}
& \overrightarrow{\mathcal{D}}=d^{(0)}-\frac{1}{1 !} d_{i_{1}}^{(1)} \zeta_{i_{1}}^{\alpha_{1}} i \vec{\partial}_{\alpha_{1}}+\frac{1}{2 !} d_{i_{1} i_{2}}^{(2)} \zeta_{i_{1}}^{\alpha_{1}} i \vec{\partial}_{\alpha_{1}} \zeta_{i_{2}}^{\alpha_{2}} i \vec{\partial}_{\alpha_{2}}-\cdots+(-)^{n} \frac{1}{n !} d_{i_{1} \cdots i_{4}}^{(n)}\left(\zeta_{i_{1}}^{\alpha_{1}} i \vec{\partial}_{\alpha_{1}}\right)^{n_{1}} \cdots\left(\zeta_{i_{4}}^{\alpha_{4}} i \vec{\partial}_{\alpha_{4}}\right)^{n_{4}} \\
& \overleftarrow{\mathcal{D}}=d^{(0)}+\frac{1}{1 !} i \overleftarrow{\partial}_{\alpha_{1}} \zeta_{i_{1}}^{\alpha_{1}} d_{i_{1}}^{(1)}+\frac{1}{2 !} i \overleftarrow{\partial}_{\alpha_{1}} \zeta_{i_{1}}^{\alpha_{1}} i \overleftarrow{\partial}_{\alpha_{2}} \zeta_{i_{2}}^{\alpha_{2}} d_{i_{1} i_{2}}^{(2)}+\cdots+\frac{1}{n !}\left(i \overleftarrow{\partial}_{\alpha_{1}} \zeta_{i_{1}}^{\alpha_{1}}\right)^{n_{1}} \cdots\left(i \overleftarrow{\partial}_{\alpha_{4}} \zeta_{i_{4}}^{\alpha_{4}}\right)^{n_{4}} d_{i_{1} \cdots i_{4}}^{(n)}
\end{aligned}
$$

with the condition $n_{1}+\cdots+n_{4}=n$ and

$$
\begin{aligned}
d^{(0)} & :=\left.\mathcal{D}\right|_{\left\{\xi_{i_{1}}, \xi_{i_{2}}, \cdots, \xi_{i_{4}}\right\} \rightarrow 0}, \\
d_{i_{1}}^{(1)} & :=\left.\frac{\partial}{\partial \xi_{i_{1}}} \mathcal{D}\right|_{\left\{\xi_{i_{1}}, \xi_{i_{2}}, \cdots, \xi_{i_{4}}\right\} \rightarrow 0}, \\
\cdots & , \\
d_{i_{1} i_{2} i_{3} i_{4}}^{(n)} & :=\left.\frac{\partial^{n}}{\partial \xi_{i_{1}}^{n} \partial \xi_{i_{2}}^{n_{2}} \cdots \partial \xi_{i_{4}}^{n}} \mathcal{D}\right|_{\left\{\xi_{i_{1}}, \xi_{i_{2}}, \cdots, \xi_{i_{4}}\right\} \rightarrow 0} .
\end{aligned}
$$

The pairs between Latin and between Greek indices in above equations denote the summation over the multiple variables $\xi_{i}(i=$ $1,2,3,4)$ and over the 4-coordinates, respectively. In order to simplify the derivations in the following appendices, we skip the summation over the multiple variables.

For the partial derivative of the Lagrangian density with respect to the spinor field $\bar{\Psi}$ only the zero-order terms in Eqs. (C2,C3) contribute. Therefore we obtain in detail

$$
\begin{aligned}
\frac{\partial \mathcal{L}}{\partial \bar{\Psi}}=\frac{1}{2} \gamma_{\mu} i \partial^{\mu} \psi-m \Psi & +\frac{1}{2} g_{\sigma} \sigma\left[d^{(0)} \Psi+\overrightarrow{\mathcal{D}} \Psi\right]-\frac{1}{2} g_{\omega} \omega^{\mu}\left[\gamma_{\mu} d^{(0)} \Psi+\gamma_{\mu} \overrightarrow{\mathcal{D}} \Psi\right]-\frac{1}{2} g_{\rho} \vec{\rho}^{\mu}\left[\gamma_{\mu} \vec{\tau} d^{(0)} \Psi+\gamma_{\mu} \vec{\tau} \overrightarrow{\mathcal{D}} \Psi\right] \\
+ & \frac{1}{2} g_{\delta} \vec{\delta}\left[\vec{\tau} d^{(0)} \Psi+\overrightarrow{\mathcal{D}} \vec{\tau} \Psi\right]
\end{aligned}
$$

and similar for the first-order derivative with respect to the spinor field $\Psi$

$$
\begin{aligned}
\frac{\partial \mathcal{L}}{\partial \Psi}=-\frac{1}{2} \gamma_{\mu} i \partial^{\mu} \bar{\psi}-m \bar{\Psi} & +\frac{1}{2} g_{\sigma}\left[\bar{\Psi} \overleftarrow{\mathcal{D}} \sigma+\bar{\Psi} d^{(0)} \sigma\right]-\frac{1}{2} g_{\omega} \omega^{\mu}\left[\bar{\Psi} \overleftarrow{\mathcal{D}} \gamma_{\mu}+\bar{\Psi} \gamma_{\mu} d^{(0)}\right]-\frac{1}{2} g_{\rho} \vec{\rho}^{\mu}\left[\bar{\Psi} \overleftarrow{\mathcal{D}} \gamma_{\mu} \vec{\tau}+\bar{\Psi} \gamma_{\mu} \vec{\tau} d^{(0)}\right] \\
& +\frac{1}{2} g_{\delta} \vec{\delta}\left[\vec{\tau} \bar{\Psi} \overleftarrow{\mathcal{D}}+\vec{\tau} \bar{\Psi} d^{(0)}\right]
\end{aligned}
$$

Concerning the partial derivatives with respect to the first-order spinor fields $\partial_{\alpha} \bar{\Psi}$ and $\partial_{\alpha} \Psi$ only the first-order derivative terms in Eqs. (C2,C3) contribute and we get

$$
\begin{aligned}
& \frac{\partial \mathcal{L}}{\partial\left(\partial_{\alpha_{1}} \bar{\Psi}\right)}=-\frac{1}{2} \gamma^{\alpha_{1}} i \psi+\frac{1}{2} g_{\sigma} d^{(1)} i \zeta^{\alpha_{1}} \Psi \sigma-\frac{1}{2} g_{\omega} d^{(1)} i \zeta^{\alpha_{1}} \gamma^{\mu} \Psi \omega_{\mu}-\frac{1}{2} g_{\rho} d^{(1)} i \zeta^{\alpha_{1}} \gamma^{\mu} \vec{\tau} \Psi \vec{\rho}_{\mu}+\frac{1}{2} g_{\delta} d^{(1)} i \zeta^{\alpha_{1}} \vec{\tau} \Psi \vec{\delta} \\
& \frac{\partial \mathcal{L}}{\partial\left(\partial_{\alpha_{1}} \Psi\right)}=\frac{1}{2} i \bar{\psi} \gamma^{\alpha_{1}}-\frac{1}{2} g_{\sigma} \sigma \bar{\Psi} i \zeta^{\alpha_{1}} d^{(1)}+\frac{1}{2} g_{\omega} \omega_{\mu} \bar{\Psi} \gamma^{\mu} i \zeta^{\alpha_{1}} d^{(1)}+\frac{1}{2} g_{\rho} \vec{\rho} \mu \bar{\Psi} \vec{\tau} \gamma^{\mu} i \zeta^{\alpha_{1}} d^{(1)}-\frac{1}{2} g_{\delta} \vec{\delta} \vec{\tau} i \zeta^{\alpha_{1}} d^{(1)}
\end{aligned}
$$


In a similar way as above one evaluates the derivatives of the Lagrangian density with respect to the second-order partial derivatives of the Dirac spinors. In this case obviously the second-order derivative terms in Eqs. (C2,C3) are of relevance, and the result reads

$$
\begin{aligned}
\frac{\partial \mathcal{L}}{\partial\left(\partial_{\alpha_{1} \alpha_{2}} \bar{\Psi}\right)} & =\frac{1}{2} g_{\sigma} d^{(2)} \frac{1}{2 !} i \zeta^{\alpha_{1}} i \zeta^{\alpha_{2}} \Psi \sigma-\frac{1}{2} g_{\omega} d^{(2)} \frac{1}{2 !} i \zeta^{\alpha_{1}} i \zeta^{\alpha_{2}} \gamma^{\mu} \Psi \omega_{\mu}-\frac{1}{2} g_{\rho} d^{(2)} \frac{1}{2 !} i \zeta^{\alpha_{1}} i \zeta^{\alpha_{2}} \gamma^{\mu} \vec{\tau} \Psi \vec{\rho}_{\mu} \\
& +\frac{1}{2} g_{\delta} d^{(2)} \frac{1}{2 !} i \zeta^{\alpha_{1}} i \zeta^{\alpha_{2}} \vec{\tau} \Psi \vec{\delta} \\
\frac{\partial \mathcal{L}}{\partial\left(\partial_{\alpha_{1} \alpha_{2}} \Psi\right)} & =\frac{1}{2} g_{\sigma} \sigma \bar{\Psi} \frac{1}{2 !} i \zeta^{\alpha_{1}} i \zeta^{\alpha_{2}} d^{(2)}-\frac{1}{2} g_{\omega} \omega_{\mu} \bar{\Psi} \gamma^{\mu} \frac{1}{2 !} i \zeta^{\alpha_{1}} i \zeta^{\alpha_{2}} d^{(2)}-\frac{1}{2} g_{\rho} \vec{\rho} \mu \bar{\Psi} \vec{\tau} \gamma^{\mu} \frac{1}{2 !} i \zeta^{\alpha_{1}} i \zeta^{\alpha_{2}} d^{(2)} \\
& +\frac{1}{2} g_{\delta} \vec{\delta} \vec{\tau} \bar{\Psi} \frac{1}{2 !} i \zeta^{\alpha_{1}} i \zeta^{\alpha_{2}} d^{(2)}
\end{aligned}
$$

With the intermediate results of this appendix we can now derive the relevant equations of the NLD model, i.e., the Diracequation for the spinor field $\Psi$ in Appendix D as well as the conserved Noether current and the energy-momentum tensor in Appendix E. Furthermore, we will perform these derivations up to second order in the higher-order fields and for the isoscalar meson-nucleon interaction Lagrangians only, since the evaluation of higher-order terms and of the other meson-nucleon vertices proceeds in a similar way. We will insert then the remaining terms, i.e., the higher-order derivatives as well as all meson-nucleon contributions in the final expressions.

In the following the terms containing the derivatives of the meson fields are not shown, since, they do not contribute on the mean-field level.

\section{Appendix D: Derivation of the Dirac-equation in the NLD model}

For the derivation of the Dirac-equation we start with the Euler-Lagrange equations of motion, Eq. (7), which read as

$$
\frac{\partial \mathcal{L}}{\partial \varphi_{r}}+\sum_{i=1}^{n}(-)^{i} \partial_{\alpha_{1} \cdots \alpha_{i}} \frac{\partial \mathcal{L}}{\partial\left(\partial_{\alpha_{1} \cdots \alpha_{i}} \varphi_{r}\right)}=0
$$

Up to second order in the partial derivatives of the spinor field $\bar{\Psi}$ they reduce to

$$
\frac{\partial \mathcal{L}}{\partial \bar{\Psi}}-\partial_{\alpha_{1}} \frac{\partial \mathcal{L}}{\partial\left(\partial_{\alpha_{1}} \bar{\Psi}\right)}+\partial_{\alpha_{1}} \partial_{\alpha_{2}} \frac{\partial \mathcal{L}}{\partial\left(\partial_{\alpha_{1}} \partial_{\alpha_{2}} \bar{\Psi}\right)}=0
$$

We insert the various partial field derivatives, Eqs. (C7), (C9) and (C11), into the second order Euler-Lagrange equations, Eq. (D2), and obtain

$$
\begin{aligned}
\gamma_{\mu} i \vec{\partial}^{\mu} \psi-m \Psi+ & \frac{1}{2} g_{\sigma} \sigma\left[d^{(0)} \Psi+\overrightarrow{\mathcal{D}} \Psi\right]-\frac{1}{2} g_{\omega} \omega^{\mu}\left[d^{(0)} \gamma_{\mu} \Psi+\gamma_{\mu} \overrightarrow{\mathcal{D}} \Psi\right] \\
& -\frac{1}{2} g_{\sigma} \sigma d^{(1)} \zeta^{\alpha_{1}} i \vec{\partial}_{\alpha_{1}} \Psi+\frac{1}{2} g_{\omega} \omega_{\mu} d^{(1)} \zeta^{\alpha} \gamma^{\mu} i \vec{\partial}_{\alpha_{1}} \Psi \\
& +\frac{1}{2} g_{\sigma} \sigma d^{(2)} \frac{1}{2 !} \zeta^{\alpha_{1}} \zeta^{\alpha_{2}} i \vec{\partial}_{\alpha_{1}} i \vec{\partial}_{\alpha_{2}} \Psi-\frac{1}{2} g_{\omega} d^{(2)} \omega_{\mu} \frac{1}{2 !} \zeta^{\alpha_{1}} \zeta^{\alpha_{2}} \gamma^{\mu} i \vec{\partial}_{\alpha_{1}} i \vec{\partial}_{\alpha_{2}} \Psi=0
\end{aligned}
$$

We rewrite Eq. (D3) such to separate the series contributions from the standard terms and obtain following expression

$$
\begin{aligned}
\left\{\gamma_{\mu} i \vec{\partial}^{\mu}-m+\right. & \frac{1}{2} g_{\sigma} \sigma \overrightarrow{\mathcal{D}}-\frac{1}{2} g_{\omega} \gamma^{\mu} \omega_{\mu} \overrightarrow{\mathcal{D}} \\
+ & \frac{1}{2} g_{\sigma} \sigma\left[d^{(0)}-d^{(1)} \zeta^{\alpha_{1}} i \vec{\partial}_{\alpha_{1}}+\frac{1}{2 !} d^{(2)} \zeta^{\alpha_{1}} i \vec{\partial}_{\alpha_{1}} \zeta^{\alpha_{2}} i \vec{\partial}_{\alpha_{2}}\right] \\
& \left.-\frac{1}{2} g_{\omega} \omega_{\mu}\left[d^{(0)}-d^{(1)} \zeta^{\alpha_{1}} i \vec{\partial}_{\alpha_{1}}+\frac{1}{2 !} d^{(2)} \zeta^{\alpha_{1}} i \vec{\partial}_{\alpha_{1}} \zeta^{\alpha_{2}} i \vec{\partial}_{\alpha_{2}}\right] \gamma^{\mu}\right\} \Psi=0
\end{aligned}
$$


In fact, if one would perform above procedure for all higher-order terms, one would obtain

$$
\begin{aligned}
& \left\{\gamma_{\mu} i \vec{\partial} \mu-m+\frac{1}{2} g_{\sigma} \sigma \overrightarrow{\mathcal{D}}-\frac{1}{2} g_{\omega} \gamma^{\mu} \omega_{\mu} \overrightarrow{\mathcal{D}}\right. \\
+ & \frac{1}{2} g_{\sigma} \sigma\left[d^{(0)}-d^{(1)} \zeta^{\alpha_{1}} i \vec{\partial}_{\alpha_{1}}+\frac{1}{2 !} d^{(2)} \zeta^{\alpha_{1}} i \vec{\partial}_{\alpha_{1}} \zeta^{\alpha_{2}} i \vec{\partial}_{\alpha_{2}}+\cdots+(-)^{n} \frac{1}{n !} d^{(n)} \zeta^{\alpha_{1}} i \vec{\partial}_{\alpha_{1}} \cdots \zeta^{\alpha_{n}} i \vec{\partial}_{\alpha_{n}}\right] \\
- & \left.\frac{1}{2} g_{\omega} \omega_{\mu}\left[d^{(0)}-d^{(1)} \zeta^{\alpha_{1}} i \vec{\partial}_{\alpha_{1}}+\frac{1}{2 !} d^{(2)} \zeta^{\alpha_{1}} i \vec{\partial}_{\alpha_{1}} \zeta^{\alpha_{2}} i \vec{\partial}_{\alpha_{2}}+\cdots+(-)^{n} \frac{1}{n !} d^{(n)} \zeta^{\alpha_{1}} i \vec{\partial}_{\alpha_{1}} \cdots \zeta^{\alpha_{n}} i \vec{\partial}_{\alpha_{n}}\right] \gamma^{\mu}\right\} \Psi=0 .
\end{aligned}
$$

The infinite series inside the brackets in Eq. (D5) add together with the non-linear terms in the first line of Eq. (D5) for each meson-nucleon vertex. One arrives to the following Dirac equation for the spinor field $\Psi$ in the NLD model

$$
\left[\gamma_{\mu} i \partial^{\mu}-g_{\omega} \gamma^{\mu} \omega_{\mu} \overrightarrow{\mathcal{D}}-g_{\rho} \gamma^{\mu} \vec{\tau} \vec{\rho}_{\mu} \overrightarrow{\mathcal{D}}-m+g_{\sigma} \sigma \overrightarrow{\mathcal{D}}+g_{\delta} \vec{\tau} \vec{\delta} \overrightarrow{\mathcal{D}}\right] \Psi=0
$$

This is the desired result, Eq. (28). Again the terms containing the derivatives of meson fields are not show in the above equation, since, they will not contribute in the final RMF expressions.

\section{Appendix E: Derivation of the Noether current in the NLD model}

As in the derivation of the Dirac equation, we consider in the following only terms up to second order in the field derivatives, and use Eqs. (C9), (C10), (C11) and (C12). We start from the general expression in Eq. (9) for the nucleonic degrees of freedom which up to second order takes the following form

$$
J^{\mu}=-i\left\{\left[\frac{\partial \mathcal{L}}{\partial\left(\partial_{\mu} \Psi\right)}-\partial_{\beta} \frac{\partial \mathcal{L}}{\partial\left(\partial_{\mu} \partial_{\beta} \Psi\right)}\right] \Psi-\bar{\Psi}\left[\frac{\partial \mathcal{L}}{\partial\left(\partial_{\mu} \bar{\Psi}\right)}-\partial_{\beta} \frac{\partial \mathcal{L}}{\partial\left(\partial_{\mu} \partial_{\beta} \bar{\Psi}\right)}\right]+\left[\frac{\partial \mathcal{L}}{\partial\left(\partial_{\mu} \partial_{\beta} \Psi\right)}\right] \partial_{\beta} \Psi-\partial_{\beta} \bar{\Psi}\left[\frac{\partial \mathcal{L}}{\partial\left(\partial_{\mu} \partial_{\beta} \bar{\Psi}\right)}\right]\right\}
$$

We rewrite now Eq. (E1) by separating the terms between the different orders in the partial derivatives (the order is labeled by a subscript)

$$
J_{\mu}=\mathcal{O}_{\mu}^{(1)}+\mathcal{O}_{\mu}^{(2)}
$$

The first-order contribution to Eq. (E2) reads as

$$
\mathcal{O}_{\mu}^{(1)}=-i\left(\frac{\partial \mathcal{L}}{\partial\left(\partial^{\mu} \Psi\right)} \Psi-\bar{\Psi} \frac{\partial \mathcal{L}}{\partial\left(\partial^{\mu} \bar{\Psi}\right)}\right)
$$

while the second-order contribution to Eq. (E2) takes the following form

$$
\mathcal{O}_{\mu}^{(2)}=-i\left[-\left(\partial_{\beta} \frac{\partial \mathcal{L}}{\partial\left(\partial^{\mu} \partial_{\beta} \Psi\right)} \Psi-\bar{\Psi} \partial_{\beta} \frac{\partial \mathcal{L}}{\partial\left(\partial^{\mu} \partial_{\beta} \bar{\Psi}\right)}\right)+\left(\frac{\partial \mathcal{L}}{\partial\left(\partial^{\mu} \partial_{\beta} \Psi\right)} \partial_{\beta} \Psi-\partial_{\beta} \bar{\Psi} \frac{\partial \mathcal{L}}{\partial\left(\partial^{\mu} \partial_{\beta} \Psi\right)}\right)\right]
$$

For the evaluation of the first-order contribution $\mathcal{O}_{\mu}^{(1)}$, Eq. (E3), we insert Eq. (C10) and its adjoint form, Eq. (C9), into Eq. (E3), and obtain

$$
\mathcal{O}_{\mu}^{(1)}=\bar{\Psi} \gamma_{\mu} \Psi-\frac{1}{2} g_{\sigma}\left[\sigma \bar{\Psi} \zeta_{\mu} d^{(1)} \Psi+\bar{\Psi} d^{(1)} \zeta_{\mu} \Psi \sigma\right]+\frac{1}{2} g_{\omega}\left[\omega_{\alpha} \bar{\Psi} \gamma^{\alpha} \zeta_{\mu} d^{(1)} \Psi+\bar{\Psi} d^{(1)} \zeta_{\mu} \gamma^{\alpha} \Psi \omega_{\alpha}\right]
$$

The derivation of the second-order contribution $\mathcal{O}_{\mu}^{(2)}$, Eq. (E4), proceeds in a similar way. We get

$$
\begin{aligned}
\mathcal{O}_{\mu}^{(2)}= & -\frac{1}{2} g_{\sigma} \sigma \bar{\Psi} i \overleftarrow{\partial}_{\beta} \frac{1}{2 !} \zeta_{\mu} \zeta^{\beta} d^{(2)} \Psi+\frac{1}{2} g_{\sigma} \bar{\Psi} d^{(2)} \frac{1}{2 !} \zeta_{\mu} \zeta^{\beta} i \vec{\partial}_{\beta} \Psi \sigma \\
& +\frac{1}{2} g_{\sigma} \sigma \bar{\Psi} \frac{1}{2 !} \zeta_{\mu} \zeta^{\beta} d^{(2)} i \vec{\partial}_{\beta} \Psi-\frac{1}{2} g_{\sigma} \bar{\Psi} d^{(2)} i \overleftarrow{\partial}_{\beta} \frac{1}{2 !} \zeta_{\mu} \zeta^{\beta} \Psi \sigma \\
& +\frac{1}{2} g_{\omega} \omega_{\delta} \bar{\Psi} i \overleftarrow{\partial}_{\beta} \frac{1}{2 !} \zeta_{\mu} \zeta^{\beta} \gamma^{\delta} d^{(2)} \Psi-\frac{1}{2} g_{\omega} \bar{\Psi} d^{(2)} \frac{1}{2 !} \zeta_{\mu} \zeta^{\beta} \gamma^{\delta} i \vec{\partial}_{\beta} \Psi \omega_{\delta} \\
& -\frac{1}{2} g_{\omega} \omega_{\delta} \bar{\Psi} \gamma^{\delta} \frac{1}{2 !} \zeta_{\mu} \zeta^{\beta} d^{(2)} i \vec{\partial}_{\beta} \Psi+\frac{1}{2} g_{\omega} \bar{\Psi} d^{(2)} i \overleftarrow{\partial}_{\beta} \frac{1}{2 !} \zeta_{\mu} \zeta^{\beta} \gamma^{\delta} \Psi \omega_{\delta}
\end{aligned}
$$


For each isoscalar meson-nucleon interaction we obtain now 4 terms, which differ between each other in the direction where the partial derivative operators act. We arrive to the following expression

$$
\begin{aligned}
\mathcal{O}_{\mu}^{(2)}= & +\frac{1}{2} g_{\sigma} \sigma \bar{\Psi} \frac{2}{2 !} \zeta_{\mu} \zeta^{\beta} d^{(2)} i \vec{\partial}_{\beta} \Psi-\frac{1}{2} g_{\sigma} \bar{\Psi} d^{(2)} i \overleftarrow{\partial}_{\beta} \frac{2}{2 !} \zeta_{\mu} \zeta^{\beta} \Psi \sigma \\
& -\frac{1}{2} g_{\omega} \omega_{\delta} \bar{\Psi} \gamma^{\delta} \frac{2}{2 !} \zeta_{\mu} \zeta^{\beta} d^{(2)} i \vec{\partial}_{\beta} \Psi+\frac{1}{2} g_{\omega} \bar{\Psi} d^{(2)} i \overleftarrow{\partial}_{\beta} \frac{2}{2 !} \zeta_{\mu} \zeta^{\beta} \gamma^{\delta} \Psi \omega_{\delta}
\end{aligned}
$$

The procedure is similar for the remaining higher-order derivative contributions. The evaluation procedure according Eqs. (E6) and (E7) for the third-order contribution, $\mathcal{O}_{\mu}^{(3)}$, would result in three terms for each vertex, for the fourth-order term, $\mathcal{O}_{\mu}^{(4)}$, in four terms for each vertex, and so forth.

Therefore, the resummation of all higher-order terms according

$$
J_{\mu}=\mathcal{O}_{\mu}^{(1)}+\mathcal{O}_{\mu}^{(2)}+\mathcal{O}_{\mu}^{(3)}+\cdots+\mathcal{O}_{\mu}^{(n)}
$$

leads to infinite series for each meson-nucleon interaction. For instance, for the scalar-isoscalar meson-nucleon interaction we get

$$
\begin{aligned}
& -\frac{1}{2} g_{\sigma} \bar{\Psi}\left[\frac{1}{1 !} d^{(1)} \zeta^{\mu}+\frac{2}{2 !} d^{(2)} i \overleftarrow{\partial}_{\alpha_{1}} \zeta^{\alpha_{1}} \zeta^{\mu}+\cdots+\frac{n}{n !} d^{(n)} i \overleftarrow{\partial}_{\alpha_{1}} \zeta^{\alpha_{1}} i \overleftarrow{\partial}_{\alpha_{2}} \zeta^{\alpha_{2}} \ldots i \overleftarrow{\partial}_{\alpha_{n-1}} \zeta^{\alpha_{n-1}} \zeta^{\mu}\right] \Psi \\
& +\frac{1}{2} g_{\sigma} \bar{\Psi}\left[-\frac{1}{1 !} \zeta^{\mu} d^{(1)}+\frac{2}{2 !} \zeta^{\mu} d^{(2)} \zeta^{\alpha_{1}} i \vec{\partial}_{\alpha_{1}}+\cdots+(-)^{n} \frac{n}{n !} \zeta^{\mu} d^{(n)} \zeta^{\alpha_{1}} i \vec{\partial}_{\alpha_{1}} \zeta^{\alpha_{2}} i \vec{\partial}_{\alpha_{2}} \cdots \zeta^{\alpha_{n-1}} i \vec{\partial}_{\alpha_{n-1}}\right] \Psi
\end{aligned}
$$

Note that the $n$-th term, $\mathcal{O}_{\mu}^{(n)}$, contains $(n-1)$ partial derivatives and that it appears $n$-times. These terms can be also resummed to infinite series. Indeed, by considering, for instance, the Taylor-expansion of the operator $\overrightarrow{\mathcal{D}}$

$$
\overrightarrow{\mathcal{D}}=1-d^{(1)} \zeta_{\alpha} i \vec{\partial}^{\alpha}+\frac{1}{2 !} d^{(2)} \zeta_{\alpha} i \vec{\partial}^{\alpha} \zeta_{\beta} i \vec{\partial}^{\beta}+\cdots
$$

we obtain for the derivative of $\overrightarrow{\mathcal{D}}$ with respect to the operator-like argument $i \vec{\partial}^{\mu}$

$$
\vec{\Omega}^{\mu}:=\frac{\partial \overrightarrow{\mathcal{D}}}{\partial\left(i \vec{\partial}_{\mu}\right)}=-d^{(1)} \zeta^{\mu}+\frac{2}{2 !} d^{(2)} \zeta^{\mu} \zeta_{\beta} i \vec{\partial}^{\beta}+\cdots
$$

In a similar way we obtain $\overleftarrow{\Omega}^{\mu}=\left(\vec{\Omega}^{\mu}\right)^{\dagger}$. As in the case of the scalar operators $\overrightarrow{\mathcal{D}}$ and $\overleftarrow{\mathcal{D}}$, the new vector operators $\vec{\Omega}^{\mu}$ and $\overleftarrow{\Omega}^{\mu}$ act from the right side to the spinor field $\Psi$ and from the left side to $\bar{\Psi}$, respectively. Below we will see that in the $\mathrm{RMF}$ approximation to nuclear matter both vector operators will give the derivative of the scalar operator $\mathcal{D}$ with respect to the single-particle 4-momentum $p^{\mu}$.

Collecting now all the contributions from Eqs. (E5) and (E7) under consideration of Eq. (E11) we arrive to compact forms, e.g., the scalar-isoscalar meson-nucleon vertex from Eq. (E9) can be resummed as follows

$$
\begin{aligned}
& -\frac{1}{2} g_{\sigma} \bar{\Psi}\left[\frac{1}{1 !} d^{(1)} \zeta^{\mu}+\frac{2}{2 !} d^{(2)} i \overleftarrow{\partial}_{\alpha_{1}} \zeta^{\alpha_{1}} \zeta^{\mu}+\cdots+\frac{n}{n !} d^{(n)} i \overleftarrow{\partial}_{\alpha_{1}} \zeta^{\alpha_{1}} i \overleftarrow{\partial}_{\alpha_{2}} \zeta^{\alpha_{2}} \ldots i \overleftarrow{\partial}_{\alpha_{n-1}} \zeta^{\alpha_{n-1}} \zeta^{\mu}\right] \Psi \\
& +\frac{1}{2} g_{\sigma} \bar{\Psi}\left[-\frac{1}{1 !} \zeta^{\mu} d^{(1)}+\frac{2}{2 !} \zeta^{\mu} d^{(2)} \zeta^{\alpha_{1}} i \vec{\partial}_{\alpha_{1}}+\cdots+(-)^{n} \frac{n}{n !} \zeta^{\mu} d^{(n)} \zeta^{\alpha_{1}} i \vec{\partial}_{\alpha_{1}} \zeta^{\alpha_{2}} i \vec{\partial}_{\alpha_{2}} \cdots \zeta^{\alpha_{n-1}} i \vec{\partial}_{\alpha_{n-1}}\right] \Psi \\
& =\frac{1}{2} g_{\sigma} \sigma \bar{\Psi} \vec{\Omega}^{\mu} \Psi-\frac{1}{2} g_{\sigma} \bar{\Psi} \overleftarrow{\Omega}^{\mu} \Psi \sigma .
\end{aligned}
$$

The evaluation method for the isovector channels of the NLD interaction Lagrangian proceeds in the same way. In total, including all degrees of freedom we obtain following compact expression for the conserved baryon current within the NLD formalism

$$
\begin{aligned}
J^{\mu}=\bar{\Psi} \gamma^{\mu} \Psi & -\frac{1}{2} g_{\sigma}\left[\bar{\Psi} \overleftarrow{\Omega}^{\mu} \Psi \sigma-\sigma \bar{\Psi} \vec{\Omega}^{\mu} \Psi\right]+\frac{1}{2} g_{\omega}\left[\bar{\Psi} \overleftarrow{\Omega}^{\mu} \gamma^{\alpha} \Psi \omega_{\alpha}-\omega_{\alpha} \bar{\Psi} \gamma^{\alpha} \vec{\Omega}^{\mu} \Psi\right] \\
+ & \frac{1}{2} g_{\rho}\left[\bar{\Psi} \overleftarrow{\Omega}^{\mu} \gamma^{\alpha} \vec{\tau} \Psi \vec{\rho}_{\alpha}-\vec{\rho}_{\alpha} \bar{\Psi} \vec{\tau} \gamma^{\alpha} \vec{\Omega}^{\mu} \Psi\right]-\frac{1}{2} g_{\delta}\left[\bar{\Psi} \overleftarrow{\Omega}{ }^{\mu} \vec{\tau} \Psi \vec{\delta}-\vec{\delta} \bar{\Psi} \vec{\tau} \vec{\Omega}^{\mu} \Psi\right]
\end{aligned}
$$

which obeys the continuity equation $\partial_{\mu} J^{\mu}=0$.

Now we apply the RMF approximation to the general expression for the Noether current, Eq. (E13). All bilinear operator-like terms are replaced by their expectation values relative to the nuclear matter ground state. Furthermore, we use for the spinor field 
the plane-wave ansatz, Eq. (38), in order to evaluate the operators $\overrightarrow{\mathcal{D}}, \overleftarrow{\mathcal{D}}, \vec{\Omega}^{\mu}$ and $\overleftarrow{\Omega}^{\mu}$. Taking also into account the equations $i \vec{\partial}^{\mu} \Psi=p^{\mu} \Psi$ and $\bar{\Psi} i \overleftarrow{\partial}^{\mu}=-\bar{\Psi} p^{\mu}$ the current $J^{\mu}$, Eq. (E13), takes following form in the RMF approximation

$$
J^{\mu}=\left\langle\bar{\Psi} \gamma^{\mu} \Psi\right\rangle+g_{\sigma}\left\langle\bar{\Psi}\left(\partial_{p}^{\mu} \mathcal{D}\right) \Psi\right\rangle \sigma-g_{\omega}\left\langle\bar{\Psi}\left(\partial_{p}^{\mu} \mathcal{D}\right) \gamma^{\alpha} \Psi\right\rangle \omega_{\alpha}-g_{\rho}\left\langle\bar{\Psi}\left(\partial_{p}^{\mu} \mathcal{D}\right) \gamma^{\alpha} \vec{\tau} \Psi\right\rangle \vec{\rho}_{\alpha}+g_{\delta}\left\langle\bar{\Psi}\left(\partial_{p}^{\mu} \mathcal{D}\right) \vec{\tau} \Psi\right\rangle \vec{\delta}
$$

with $\partial_{p}^{\mu}=\frac{\partial}{\partial p_{\mu}}$. This is the desired result, see Eq. (47).

The derivation of the energy-momentum tensor proceeds in the same way as for the current, therefore, we skip further derivations.

[1] B. D. Serot and J. D. Walecka, Adv. Nucl. Phys. 16, 1 (1986).

[2] H.-P. Duerr, Phys. Rev. 103, 469 (1956).

[3] J. Walecka, Annals Phys. 83, 491 (1974).

[4] B. D. Serot and J. D. Walecka, Int. J. Mod. Phys. E6, 515 (1997).

[5] E. Cooper, S. Hama, B. Clark, and R. Mercer, Phys. Rev. C47, 297 (1993).

[6] S. Hama, B. Clark, E. Cooper, H. Sherif, and R. Mercer, Phys. Rev. C41, 2737 (1990).

[7] K. Weber et al., Nucl. Phys. A539, 713 (1992).

[8] S. Typel, O. Riedl, and H. H. Wolter, Nucl. Phys. A709, 299 (2002).

[9] B. Ter Haar and R. Malfliet, Phys. Rept. 149, 207 (1987).

[10] R. Brockmann and R. Machleidt, (1996), arXiv:nuclth/9612004v1.

[11] H. Müther and A. Polls, Prog. Part. Nucl. Phys. 45, 243 (2000).

[12] W. Botermans and R. Malfliet, Phys. Rept. 198, 115 (1990).

[13] O. Buss et al., Phys. Rept. 512, 1 (2012).

[14] N. Hugenholtz and M. Van Hove, Physica XXVI, 363 (1958).

[15] J. Boguta and A. Bodmer, Nucl. Phys. A292, 413 (1977).

[16] Y. Sugahara and H. Toki, Nucl. Phys. A579, 557 (1994).

[17] R. Brockmann and H. Toki, Phys. Rev. Lett. 68, 3408 (1992).

[18] C. Fuchs, H. Lenske, and H. Wolter, Phys. Rev. C52, 3043 (1995).

[19] S. Typel and H. Wolter, Nucl. Phys. A656, 331 (1999).

[20] J. Zimanyi and S. Moszkowski, Phys. Rev. C42, 1416 (1990).

[21] S. Typel, T. v. Chossy, and H. Wolter, Phys. Rev. C67, 034002 (2003).

[22] S. Typel, Phys. Rev. C71, 064301 (2005).

[23] T. Gaitanos and M. Kaskulov, Nucl. Phys. A878, 49 (2012).

[24] T. Gaitanos, M. Kaskulov, and H. Lenske, Phys. Lett. B703, 193 (2011).

[25] T. Gaitanos, M. Kaskulov, and U. Mosel, Nucl. Phys. A828, 9 (2009).

[26] F. Özel, G. Baym, and T. Güver, Phys. Rev. D82, 101301 (2010).

[27] A. W. Steiner, J. M. Lattimer, and E. F. Brown, Astrophys. J. 722, 33 (2010).

[28] R. Machleidt, K. Holinde, and C. Elster, Phys. Rept. 149, 1 (1987).

[29] K. Erkelenz, Phys. Rept. 13, 191 (1974).

[30] V. Weisskopf, Nucl. Phys. 3, 423 (1957).

[31] B. Ter Haar and R. Malfliet, Phys. Rev. C36, 1611 (1987).

[32] O. Plohl, C. Fuchs, and E. van Dalen, Phys. Rev. C73, 014003 (2006).

[33] J. Nelder and R. Mead, Comput.J. 7, 308 (1965).

[34] K. M. Brown, SIAM Journal on Numerical Analysis 6, 560 (1969).
[35] D. John, G. David, and R. Welsch, ACM Transactions on Mathematical Software 7, 367 (1981).

[36] J. J. More, B. S. Garbow, and K. E. Hillstrom, Technical Report ANL-80-74.

[37] G. Lalazissis et al., Phys. Lett. B671, 36 (2009).

[38] G. Li, R. Machleidt, and R. Brockmann, Phys. Rev. C45, 2782 (1992).

[39] R. Brockmann and R. Machleidt, Phys. Rev. C42, 1965 (1990).

[40] T. Gross-Boelting, C. Fuchs, and A. Faessler, Nucl. Phys. A648, 105 (1999).

[41] W. D. Myers and W. Swiatecki, Annals Phys. 55, 395 (1969).

[42] W. D. Myers and W. Swiatecki, Annals Phys. 84, 186 (1974).

[43] J. Blaizot, Phys. Rept. 64, 171 (1980).

[44] D. H. Youngblood, H. L. Clark, and Y.-W. Lui, Phys. Rev. Lett. 82, 691 (1999).

[45] T. v. Chossy and W. Stocker, Phys. Rev. C56, 2518 (1997).

[46] B.-A. Li, L.-W. Chen, and C. M. Ko, Phys. Rept. 464, 113 (2008).

[47] T. Li et al., Phys. Rev. Lett. 99, 162503 (2007).

[48] P. Danielewicz, R. Lacey, and W. G. Lynch, Science 298, 1592 (2002).

[49] P. Sahu, A. Hombach, W. Cassing, and U. Mosel, Nucl. Phys. A640, 693 (1998).

[50] T. Gaitanos, C. Fuchs, H. Wolter, and A. Faessler, Eur. Phys. J. A12, 421 (2001).

[51] C. Fuchs, Prog. Part. Nucl. Phys. 56, 1 (2006).

[52] C. Fuchs, A. Faessler, E. Zabrodin, and Y.-M. Zheng, Phys. Rev. Lett. 86, 1974 (2001).

[53] C. Hartnack, H. Oeschler, and J. Aichelin, Phys. Rev. Lett. 96, 012302 (2006).

[54] C. Hartnack, H. Oeschler, Y. Leifels, E. L. Bratkovskaya, and J. Aichelin, Phys. Rept. 510, 119 (2012).

[55] T. Gaitanos et al., Nucl. Phys. A732, 24 (2004).

[56] D. Shetty and S. Yennello, Pramana 75, 259 (2010).

[57] D. Shetty, S. Yennello, and G. Souliotis, Phys. Rev. C76, 024606 (2007).

[58] M. Jaminon and C. Mahaux, Phys. Rev. C40, 354 (1989).

[59] B. Blaettel, V. Koch, and U. Mosel, Rept. Prog. Phys. 56, 1 (1993).

[60] G. Lalazissis, J. Konig, and P. Ring, Phys. Rev. C55, 540 (1997).

[61] V. Baran, M. Colonna, V. Greco, and M. Di Toro, Phys. Rept. 410, 335 (2005).

[62] W. Zuo, L. Cao, B. Li, U. Lombardo, and C. Shen, Phys. Rev. C72, 014005 (2005).

[63] E. van Dalen, C. Fuchs, and A. Faessler, Phys. Rev. C72, 065803 (2005).

[64] S. Typel, private communication (2012). 
[65] J. Boguta, Phys. Lett. B106, 250 (1981).

[66] T. Klaehn et al., Phys. Rev. C74, 035802 (2006).

[67] P. Demorest, T. Pennucci, S. Ransom, M. Roberts, and J. Hessels, Nature 467, 1081 (2010).

[68] S. Thorsett and D. Chakrabarty, Astrophys. J. 512, 288 (1999).

[69] G. Baym, C. Pethick, and P. Sutherland, Astrophys. J. 170, 299 (1971).

[70] N. Glendenning, General relativity and compact stars
(Springer, 2005).

[71] I. Sagert, M. Hempel, C. Greiner, and J. Schaffner-Bielich, Eur. J. Phys. 27, 577 (2006).

[72] F. Weber, Prog. Part. Nucl. Phys. 54, 193 (2005).

[73] J. Lattimer and M. Prakash, Science 304, 536 (2004).

[74] J. M. Lattimer and M. Prakash, Phys. Rept. 442, 109 (2007).

[75] P. Freire et al., Monthly Notices of the Royal Astronomical Society, 412: 27632780. doi: 10.1111/j.1365-2966.2010.18109.x, arXiv:1011.5809v1 [astro-ph.GA]. 